\title{
Evidence supporting a critical contribution of intrinsically disordered regions to the biochemical behavior of full-length human HP1 $\gamma$
}

\author{
Gabriel Velez ${ }^{1,2,3,4}$ - Marisa Lin ${ }^{1,2,3}$ - Trace Christensen ${ }^{1,2,3}$ - William A. Faubion ${ }^{1,2,3}$. \\ Gwen Lomberk $^{1,2,3} \cdot$ Raul Urrutia ${ }^{1,2,3}$ (1)
}

Received: 5 July 2015 / Accepted: 22 November 2015 / Published online: 17 December 2015

(C) The Author(s) 2015. This article is published with open access at Springerlink.com

\begin{abstract}
HP1 $\gamma$, a non-histone chromatin protein, has elicited significant attention because of its role in gene silencing, elongation, splicing, DNA repair, cell growth, differentiation, and many other cancer-associated processes, including therapy resistance. These characteristics make it an ideal target for developing small drugs for both mechanistic experimentation and potential therapies. While high-resolution structures of the two globular regions of HP $1 \gamma$, the chromo- and chromoshadow domains, have been solved, little is currently known about the conformational behavior of the full-length protein. Consequently, in the current study, we use threading, homology-based molecular modeling, molecular mechanics calculations, and molecular dynamics simulations to develop models that allow us to infer properties of full-length $\mathrm{HP} 1 \gamma$ at
\end{abstract}

Electronic supplementary material The online version of this article (doi:10.1007/s00894-015-2874-z) contains supplementary material, which is available to authorized users.

Gwen Lomberk

lomberk.gwen@mayo.edu

$\triangle$ Raul Urrutia

urrutia.raul@mayo.edu

1 Laboratory of Epigenetics and Chromatin Dynamics, Gastroenterology Research Unit, Department of Biochemistry and Molecular Biology, Mayo Clinic, 200 First Street SW, Guggenheim 10, Rochester, MN 55905, USA

2 Laboratory of Epigenetics and Chromatin Dynamics, Gastroenterology Research Unit, Department of Biophysics, Mayo Clinic, Rochester, MN, USA

3 Laboratory of Epigenetics and Chromatin Dynamics, Gastroenterology Research Unit, Department of Medicine, Mayo Clinic, Rochester, MN, USA

4 Medical Scientist Training Program, University of Iowa, Iowa City, IA, USA an atomic resolution level. HP1 $\gamma$ appears as an elongated molecule in which three Intrinsically Disordered Regions (IDRs, 1, 2, and 3) endow this protein with dynamic flexibility, intermolecular recognition properties, and the ability to integrate signals from various intracellular pathways. Our modeling also suggests that the dynamic flexibility imparted to HP1 $\gamma$ by the three IDRs is important for linking nucleosomes with PXVXL motif-containing proteins, in a chromatin environment. The importance of the IDRs in intermolecular recognition is illustrated by the building and study of both IDR2 HP1 $\gamma$-importin- $\alpha$ and IDR 1 and IDR2 HP1 $\gamma$-DNA complexes. The ability of the three IDRs for integrating cell signals is demonstrated by combined linear motif analyses and molecular dynamics simulations showing that posttranslational modifications can generate a histone mimetic sequence within the IDR 2 of HP1 $\gamma$, which when bound by the chromodomain can lead to an autoinhibited state. Combined, these data underscore the importance of IDRs 1, 2, and 3 in defining the structural and dynamic properties of $\mathrm{HP} 1 \gamma$, discoveries that have both mechanistic and potentially biomedical relevance.

Keywords HP1 $\cdot \mathrm{HP} 1 \gamma \cdot \mathrm{CBX} 3 \cdot$ Molecular modeling · Molecular dynamics $\cdot$ Epigenetics $\cdot$ Chromatin

\section{Introduction}

The heterochromatin protein 1 (HP1) family of histone mark readers, the focus of the current study, was one of the first types of chromatin regulators to be identified $[1,2]$. This family of proteins participates in evolutionarily conserved processes in organisms ranging from early eukaryotes to humans $[2,3]$. Human cells produce three different HP1 protein isoforms, HP1 $\alpha$ (CBX5), HP1 $\beta$ (CBX1), and HP1 $\gamma$ (CBX3), 
which regulate the expression of entire networks of genes that are critical for normal embryonic development and the maintenance of most homeostatic processes, including cell cycle control, proliferation, apoptosis, differentiation, and DNA damage response $[2,4]$. In addition, the expression and deregulation of HP1-mediated processes associate with the development, spreading, and prognosis of several cancers [4]. Consequently, better understanding of the biochemical properties of HP1 proteins has both biological and medical implications.

The current work represents an extension of work in our laboratory, which seeks to understand the biological and pathobiological roles of HP1 $\gamma$. Early biochemical studies revealed that HP1 $\gamma$ recognizes and binds specific di- and trimethylated forms of histones (K9H3 and $\mathrm{K} 26 \mathrm{H} 1)$ and translates this biochemical information into a defined pattern of gene expression [5-7]. The ability of HP1 $\gamma$ to recognize this mark was subsequently mapped to a small region within the $\mathrm{N}$-terminal domain, known as chromodomain [8]. In addition, HP1 $\gamma$ uses this chromodomain to recruit the related histone methyltransferases, G9a and GLP, which write dimethylated K9 histone marks as part of a positive-feedback loop that leads to increased concentration of reader-writer complexes on specific genomic regions where they are needed to regulate gene expression [3]. G9a and GLP have the ability to automethylate at an internal K-containing peptide, which mimics methylated-histones (histone mimicry) [9]. HP1 $\gamma$ also recruits an additional histone methyltransferase protein, SUV39H1, in a manner that is independent of its methylation status, but rather contains a specific linear motif with a PXVXL consensus sequence [10]. For recognizing and binding the PXVXL motif, HP1 $\gamma$ must first form homodimers or heterodimers with HP1 $\alpha$ or HP1 $\beta[3,10]$. Dimerization and PXVXL recognition, which is imparted to HP1 $\gamma$ by its N-terminal chromoshadow domain, recruits additional chromatin regulators that may impart further instructions for the regulation of genomic and epigenomic functions $[3,10]$. Thus, due to the functional importance of both the chromo- and chromoshadow domains, structural studies have begun to focus on deciphering the biophysical properties that determine their function, in the hope that this knowledge may aid in the development of drugs for manipulating HP1 $\gamma$-mediated processes in experimental and therapeutic settings [3].

Several laboratories have focused on studying the function of less well-characterized regions of the HP1 $\gamma$ molecules, namely the most $\mathrm{N}$ - and $\mathrm{C}$-terminal regions located between the chromo and chromoshadow domains. Unfortunately, in this regard, no NMR or X-ray crystallographic studies have yet yielded any useful information regarding the properties of these less-known domains [11, 12]. Therefore, there is a need for a better understanding of the structure and biophysical behavior of full-length human HP1 $\gamma$ by assigning biophysical properties of those domains for which data at the atomic resolution is lacking, establishing their role in molecular connectivity and flexibility as well as intermolecular interactions. Consequently, using a combination of structural bioinformatics, molecular modeling methods, and molecular dynamics approaches, we here report that HP1 $\gamma$ is an elongated molecule, in which three Intrinsically Disordered Regions (IDRs, 1, 2, and 3) endow this protein with dynamic flexibility, intermolecular recognition properties, and the ability to integrate signals from various intracellular pathways. Our models and the inferences derived from them integrate, complement, explain, and extend available experimental data, providing new insights that can serve as the structural rationale for future experimentations and drug design.

\section{Materials and methods}

Generation of a structural model for full-length HP1 $\gamma$ To evaluate the disorder probability of the N- (Met1 - Val31) and C-terminal (Arg171 - Gln183) regions as well as the HP1 $\gamma$ linker (Ala82 - Arg115), we used a meta-prediction approach that integrated the data from PrDOS [13], metaPrDOS [14], POODLE [15], DISpro [16], DisEMBL [17], IUPred [18], PONDR-FIT [19], PreDisorder [20], OnD-CRF [21], RONN [22], FoldIndex [23], DISOclust [24], and GlobPlot2 [25]. As a negative control, we subjected the sequence of an ordered alpha helix from HP1 $\gamma$ (PQIVIAFYEER; residues 161-171) to the same analysis. The N-terminal domain of the HP1 $\gamma$ isoforms were modeled using the threading algorithms LOMETS [26] and MUSTER [27]. The C-terminal regions were modeled as disordered regions using homology-based modeling with the mouse structure of HP1 $\beta$ (PDB: 3Q6S) [28]. In addition, a final homology-based model of the HP1 $\gamma$ linker regions were built using the solved structure of the bipartite NLS from nucleophosmin (PDB: 1PJN) in MODELLER [28]. All of these models congruently predict that these HP1 $\gamma$ sequences constitute Intrinsically Disorder Regions, herein called IDR1, IDR2, and IDR3. Full models of the HP1 isoforms (PDB: 1GUW and 3Q6S for HP1 $\beta$; 3KUP and 3DM1 for HP1 $\gamma$; as well as 3I3C and 3FDT for HP1 $\alpha$ ) were constructed using the peptide bonding function of the Builder feature of Discovery Studio 4.1 [29] to establish the following molecular connectivity IDR1-chromodomainIDR2-chromoshadow domain-IDR3 (Fig. 1a). Biophysical properties of this model (i.e., volume, electrostatics, etc.) were calculated using VADAR [30] and the $3 \mathrm{~V}$ Volume Calculator [31].

Modeling of HP1 complexes The HP1 $\gamma-\mathrm{HP} 1 \gamma$ homodimer and heterodimers with HP1 $\alpha$ and HP1 $\beta$ were docked by homology using the structure of the chromoshadow domain of the mouse HP1 $\alpha$ and HP1 $1 \beta$ (PDB: 1GUW and 3Q6S for HP1 $\beta$; 3KUP and 3DM1 for HP1 $\gamma$; as well as 3I3C and 3FDT for HP1 $\alpha$ ). The three-dimensional complex structure 
A

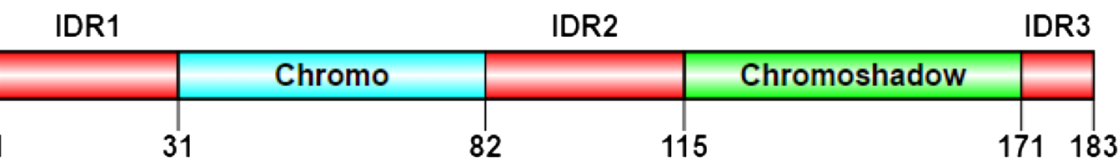

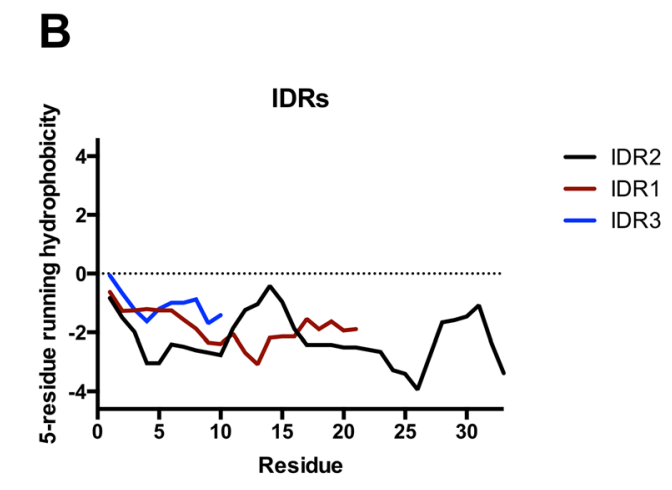

C

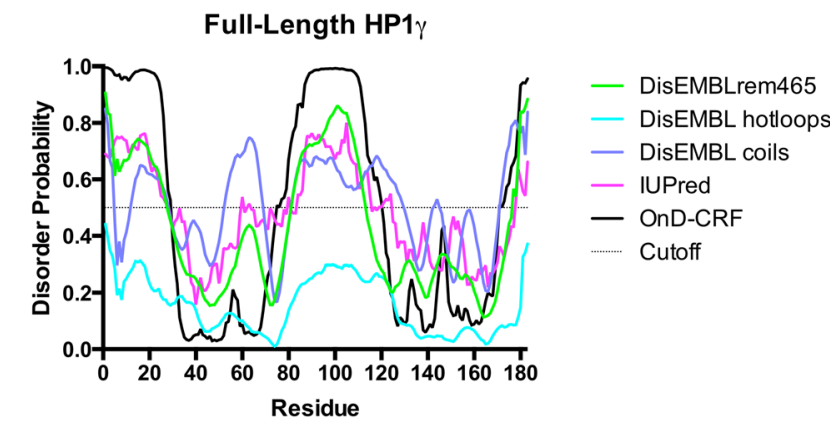

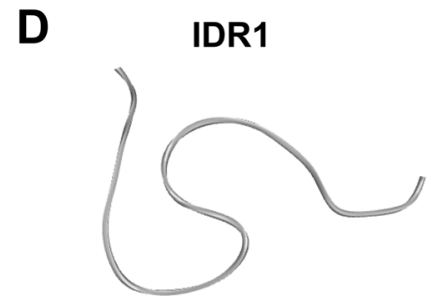

E
IDR2

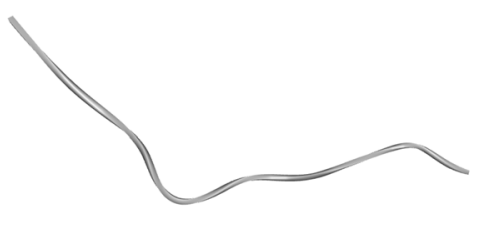

$\mathbf{F}$
IDR3
Fig. 1 Structural bioinformatics reveal the HP1 $\gamma$ IDR regions to have a high propensity toward disorder. a Linear domain graph showing amino acid positions for IDR1 (Met1 - Val31), IDR2 (Ala82 - Arg115), and IDR3 (Arg171 - Gln183). b Hydrophobicity plot reveals the IDRs have a high polar to hydrophobic ratio of residues, a feature that characterizes intrinsically disordered proteins. c Disorder meta-prediction for fulllength HP1 $\gamma$ reveals the IDRs to have a higher propensity toward disorder, while the chromo and chromoshadow domain are predicted to be ordered regions of the protein. Disorder probability values above the cut-off value of 0.5 are considered to be disordered. d Structure predictions of IDR $1 \mathbf{e}$, IDR2, and IDR $3 \mathbf{f}$ predicted by threading algorithms show that threading cannot model these regions in any secondary or tertiary conformation

Dock uses a nonspecific B-DNA model to probe the binding site on a 3D model of a protein that is known to bind DNA, but for which the specific contacts are unknown. Using the structure of a DNA binding protein as input, the method first automatically generated an ensemble of protein-DNA complexes obtained by rigid-body docking with nonspecific canonical BDNA molecules [33]. Models were subsequently selected by clustering and ranking them according to their DNA-protein interfacial energies [33].

Molecular dynamics (MD) simulations The MD simulations of HP1 $\gamma$ and its complexes were performed using the all-atom force field in CHARMm c36b2 at a temperature of $300 \mathrm{~K}$ (NVT ensemble) [35]. The molecule was first energyminimized using a two-step protocol of steepest descent and conjugated gradients. All of these steps were done using the SHAKE procedure [36]. A distance-dependent dielectrics implicit solvent model was used with a dielectric constant of 80 
and a $\mathrm{pH}$ of 7.4. Using the same procedure, additional MD simulations were performed on models of HP1 complexes and on HP1 $\gamma$ mutants. In order to better approximate experimental conditions, additional simulations were run using generalized born (GB) implicit solvation with single switching and a $\mathrm{NaCl}$ concentration of $150 \mathrm{mM}$ [37]. Studies on the flexibility of HP1 $\gamma$ required performance of two simulations, one at $100 \mathrm{ps}$ and another at $2 \mathrm{~ns}$.

\section{Linear motif analysis for post-translational modifications,} protein-protein interaction domains, protein-protein interaction motifs The presence of a nuclear localization signal (NLS) was derived by combining linear motifs analysis using PsortII, confirming the similarity with other NLSs by virtual peptide display method using Prints [38]. The potential of the IDR1 and IDR2 for binding to DNA was predicted using DPBind [39]. Prediction of post-translational modification sites on the CBX isoforms was performed by compiling and statistically scoring linear motifs for phosphorylation, acetylation, methylation, ubiquitination, and sumoylation as predicted by 20 different software programs. The software used to predict phosphorylation were NetPhosK 1.0 [40], NetPhos 2.0 [40], Kinasephos 2.0 [41], DIPHOS [42], PhosphoSVM [43], Scansite, Musite [44], and PPSP [45]. Acetylation sites were predicted using PAIL [46], ASEB [47], BRABSB-PHKA [48], LysAcet [49], and LAceP [50]. Methylation sites were predicted using BPB-PPMS [51] and MASA [52]. Ubiquitination sites were predicted using BDM-PUB [53], CKSAAP UbSite [54], and UbPred [55]. Sumoylation sites were predicted using GPS-SUMO [56] and SUMOplot (http:// www.abgent.com/sumoplot/). Results from these predictions were then compiled and statistically scored to assign specificity potential to sites that were predicted to undergo modification in HP1 proteins. Briefly, for each distinct software, we considered sites for which the prediction score was above the cut-off that had been derived using a training set of modified sequences that have been experimentally validated. Subsequently, we developed a meta-prediction score (MPS) by assigning a maximum score of 1 to sites that were predicted by all of the programs cited. Scores for other programs were numerically expressed relative to this maximum score. Results of these predictions were then compared to experimentally validated sites listed in PhosphositePlus [57] and PHOSIDA [58] databases to define whether all predicted sites have also been found in large-scale OMICs analyses.

\section{Immunoprecipitation of HP1 $\gamma$ complexes and mass spec-} trometry Subconfluent HeLa cells were lysed and immunoprecipitation of HP1 $\gamma$ was performed using the Pierce Crosslink Magnetic IP/Co-IP Kit according to the manufacturer's instructions. HP1 $\gamma$ antibody (Abcam) was cross-linked to the Protein $\mathrm{A} / \mathrm{G}$ magnetic beads using disuccinimidyl suberate (DSS) to minimize IgG contamination in the final elution. The immunoprecipitated HP1 $\gamma$ complexes were resolved on a 4-15\% Criterion Tris- $\mathrm{HCl}$ polyacrylamide gel (Bio-Rad) and stained with Bio-Safe Coomassie Stain (BioRad) according to the manufacturer's recommendations. Subsequently, bands were selected for excision and processed for nano high-pressure liquid chromatography electrospray tandem mass spectrometry (nano-LC-ESI-MS/MS) by the Mayo Medical Genome Facility Proteomics Core.

Electron microscopy For visualizing the shape and contour of the HP1 $\gamma$ dimer, we produced and purified an N-terminal $6 \times$ His-tagged recombinant form of this protein using the $\mathrm{pET}$ vector system (Novagen, CA). The HP1 $\gamma$-encoding plasmid was grown in DE3 BL21 bacteria cells overnight and induced with $0.5 \mathrm{mM}$ IPTG for $90 \mathrm{~min}$ at $32{ }^{\circ} \mathrm{C}$. The recombinant protein was purified using the Thermo Scientific HisPur Cobalt Resin Kit according to the manufacturer's instructions. Protein was dialyzed overnight and concentrated to a final concentration of $1 \mathrm{mg} / \mathrm{ml}$. For visualization at the electron microscopy level, $10 \mu \mathrm{l}$ of the purified protein solution was placed on the surface of glow-discharged formvar carboncoated grids. After $30 \mathrm{~s}$, the grids were blotted and stained for $30 \mathrm{~s}$ in $1 \%$ uranyl acetate. Micrographs were acquired using a JEOL, JEM-1400Plus TEM at $80-\mathrm{kV}$ accelerating voltage, equipped with a Gatan Orius 832 camera.

\section{Results}

\section{Building a high-resolution molecular model of full-length human HP1 $\gamma$}

We sought to build a model to enhance our understanding of the structure and molecular dynamics of the human full-length HP1 $\gamma$. The goal of our study was to use Short Linear Motifs (SLiMs) algorithms, homology modeling, threading, in silico mutations, docking, and molecular dynamics simulations to infer biochemical and biophysical information contained particularly within those regions of the protein for which the structure has not been determined. These regions, which together encompass $41.5 \%$ of the protein, correspond to the 31 a.a. N-terminal and 12 a.a. C-terminal tail, as well as the 33 a.a. peptide that links the two known globular domains. Several observations led to modeling these regions of HP1 $\gamma$ as Intrinsically Disordered Regions (IDRs) 1, 2, and 3 (Fig. 1a), a fact that subsequent MD simulations later demonstrated. Initially, hydropathic analyses, shown in Fig. 1b, indicated that these regions display a high polar-to-hydrophobic ratio of residues, a characteristic of Intrinsically Disordered Protein Regions [59]. Furthermore, several order-to-disorder prediction algorithms, such as PrDOS [13], metaPrDOS [14], POODLE [15], DISpro [16], DisEMBL [17], IUPred [18], PONDR-FIT [19], PreDisorder [20], OnD-CRF [21], RONN [22], 
FoldIndex [23], DISOclust [24], and GlobPlot2 [25], revealed a large propensity for each of the three regions to remain unfolded as an IDR in solution (Fig 1c; Supplementary Fig. 1a-c). As a negative control, we performed the same disorder meta-prediction on a helical region of the HP1 $\gamma$ chromoshadow domain (PQIVIAFYEER; residues 161171). The results of this meta-prediction show that most of this region is ordered, as opposed to the IDRs (Supplementary Fig. 1e). Homology-based modeling of the HP1 $\gamma$ IDR2 domain, using the Xenopus laevis N1N2 phosphoprotein structure as a template (PDB: 1PJN), also indicated its tendency to adopt a random coil conformation (Fig. 1e). We chose N1N2 as a template since it was used in previous structural studies to determine the specificity of $\alpha$-importin for a variety of nuclear localization signal sequences [32]. The structure of the Nterminal tail (IDR1), also as a random coil, was derived from threading results (Fig. 1d), which were congruent with the predictions of disorder (Fig. 1c; Supplementary Fig. 1a-c). Similar random coil assignments to the structure of the HP1 $\gamma$ linker region (IDR2) and the C-terminal tail (IDR3) were obtained by threading (Fig. 1d-f) and were congruent with predictions of disorder (Supplementary Fig. 1a-c).

Thus, together, using these collective inferences, we built the final model of the full-length HP1 $\gamma$ monomer by joining all of these fragments using the bonding function of Builder (Fig. 2a) [29]. A model for the HP1 $\gamma$-HP1 $\gamma$ homodimer was built by docking the monomer using homology-based rules derived from evolutionarily conserved chromoshadow domains (overall identity of 74.6 and $100 \%$ in the residues used for docking; Fig. 2b; PDB: 3DM1). The models were then refined, first by appropriate energy minimization (see Materials and methods) using a harmonic restraint, with a scaled force constant of $10 \mathrm{kcal} / \mathrm{mol}$, on both globular domains. This minimization step was repeated after removal of the harmonic restraint. To estimate the quality of the monomer model, we generated Ramachandran plots (Psi vs. Phi angles plot) using PROCHECK [60], which revealed that $97 \%$ of residues were in favored and allowed regions (Fig. 2c). Taking into consideration that the globular domains are derived from high-resolution structures (PDB: 3KUP and 3DM1) as well as the congruency of many approaches that model the rest of the protein as IDRs, we believe that this model is highly reliable and useful.

\section{Structural and dynamics properties of the full-length HP1 $\gamma$ molecule}

In order to assess the structural and dynamic properties of our full-length model, we first calculated the length of HP1 $\gamma$, as modeled in its most extended conformation possible, using the Distance Monitor feature of Discovery Studio [29]. We computed the total comparative length of HP1 $\gamma$ with similar models built for HP1 $\alpha$ and HP1 $\beta$ by adding the length of the chromodomain, chromoshadow domain, and IDRs, as

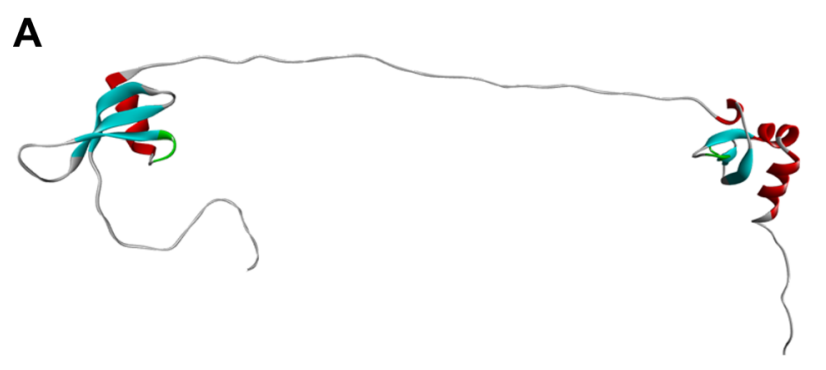

C

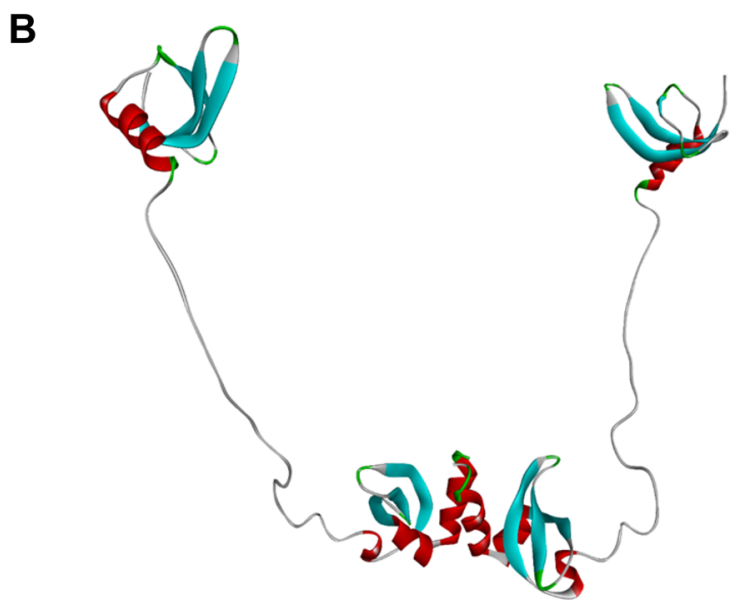

Fig. 2 Constructed models of the HP1 $\gamma$ monomer and homodimer. a Full-length model of HP1 $\gamma$ was generated by joining the globular domains (CD and CSD) with the IDRs using the Builder function in

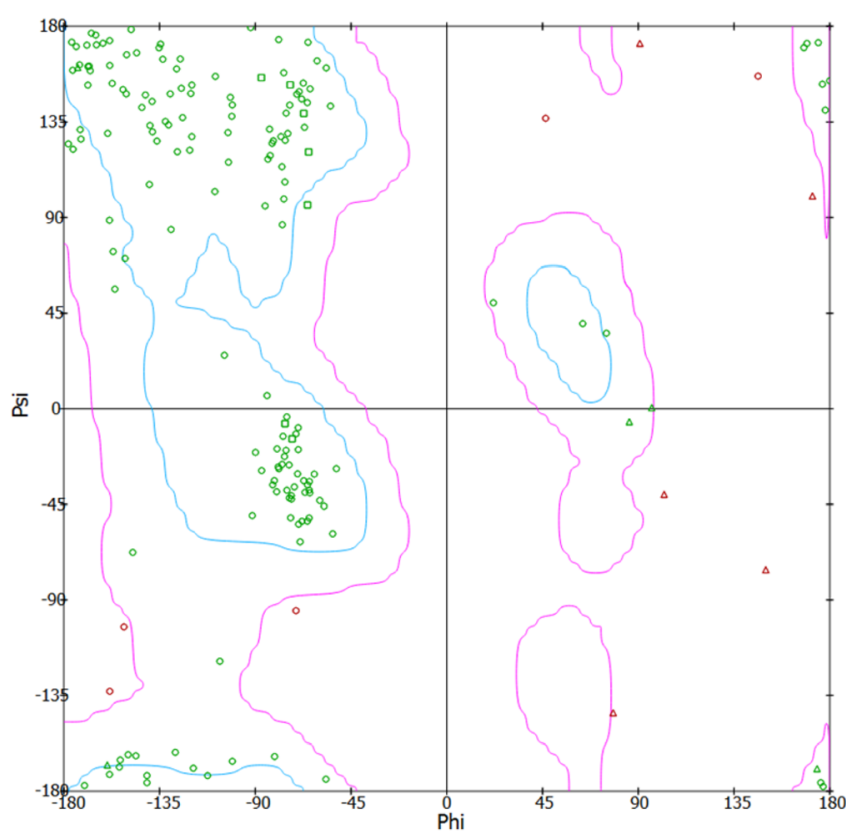

Discovery Studio Client 4.0. b Constructed model of the HP1 $\gamma$ homodimer. c Ramachandran plot reveals $97 \%$ of the residues for this model to be in favored or allowed regions 
well as the Connolly surface, which illustrates the solvent accessibility of the molecules (accessible surface area, ASA). Other comparative properties of these HP1 monomers, including volume, surface area, sphericity, center of mass, solvation energy, and electrostatic potential, were measured using VADAR [30] and the $3 \mathrm{~V}$ Volume Calculator [31]. A comparison of these properties is listed in Supplementary Table 1. A detail of the homodimerization interface for the HP1 $\gamma$ homodimer, as observed in our system, is also given in the supplementary material (Supplementary Table 2). Experimentally, we found that a model for the HP1 $\gamma$ homodimer fits nicely with the shape of HP1 $\gamma$ particles imaged using negative staining electron microscopy (Fig. 3a-c), which considerably resembled electron microscopy recently reported for the yeast HP1 $\gamma$ ortholog, SWI6 [9] as well as SAXS experiments on HP1 $\beta$ [61].

Subsequently, we performed a conformational search that might reflect the biophysical behavior of HP1 $\gamma$ using molecular dynamics simulations. Figure 4a represents an assemblage of conformers obtained during a short MD simulation. These initial simulations were run with implicit solvent models due to the computational expense of explicit solvent models. To address this issue, we ran additional MD simulations using a generalized born (GB) model with single switching to allow for simulation under a better approximation of experimental conditions $(150 \mathrm{mM} \mathrm{NaCl})$. After $2 \mathrm{~ns}$ of this simulation, the molecule had completely coiled on itself and moved freely with its three IDRs widely sampling the conformation space (Fig. 4b). RMSD and RMSF calculations provided a comparative numerical representation of the flexibility and mobility of the different domains across simulation time. The HP1 $\gamma$ IDRs displayed the highest RMSF values, suggesting that they had the highest degree of flexibility. These numerical results were consistent with the visual data provided by the conformational sampling (Fig. 4c-d) Radius of gyration calculations revealed a repetition of this movement across simulation time (Fig. 4e). Thus, the highly flexible IDR2 endows HP1 $\gamma$ with the ability to shorten its length very rapidly to adopt a final stable conformation where both globular domains come into close proximity with each other (Fig. 4a-b). Our short MD simulations display that HP1 $\gamma$ can sample a wide conformational space by populating an extended ensemble. Longer MD simulations, performed at $2 \mathrm{~ns}$ and $10 \mathrm{~ns}$, both reveal that due to the high flexibility of the hinge region, the two globular domains, $\mathrm{CD}$ and $\mathrm{CSD}$, come close together with time but do not make direct contact, a fact that would facilitate the spatial search by the two domains for their binding partners (Supplementary Fig. 2). Notably, a similar behavior for HP1 $\beta$ and the yeast HP1 protein SWI6 has been recently observed using experimental techniques. This behavior by both isoforms is likely to be important for HP1 molecules to bridge nearby nucleosomes to form heterochromatin

A
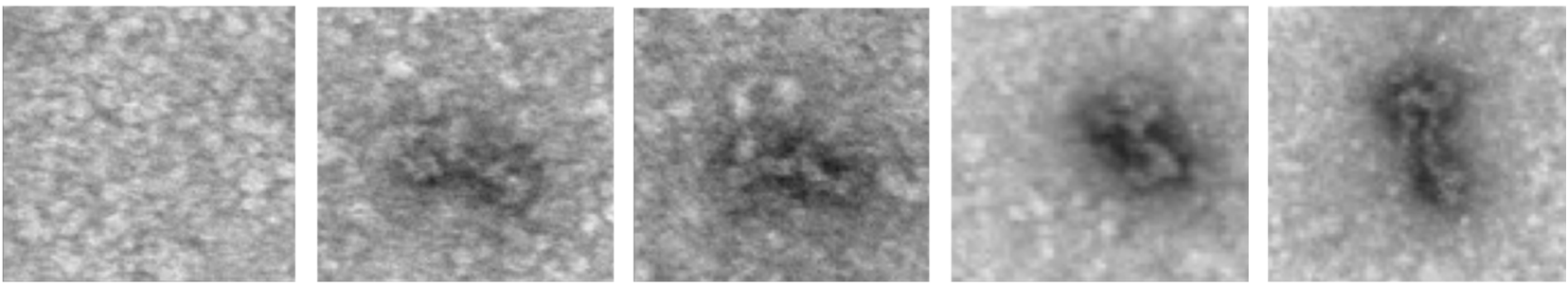

B

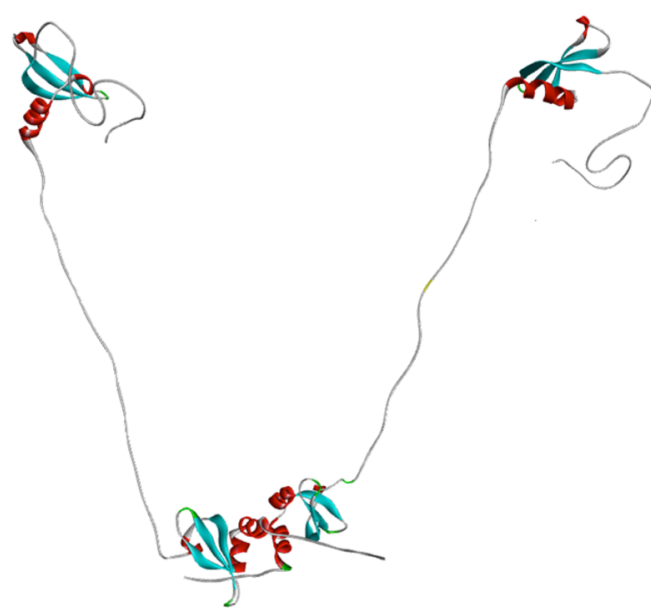

Fig. 3 Electron microscopy validates the shape of the HP1 $\gamma$ homodimer. a Electron microscopy (EM) images of purified HP1 $\gamma$. b Homologybased model of the HP1 $\gamma$ homodimer. c Superimposition of the
C

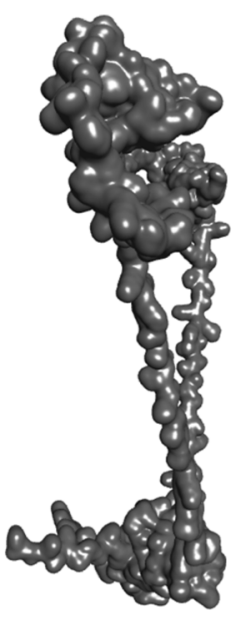

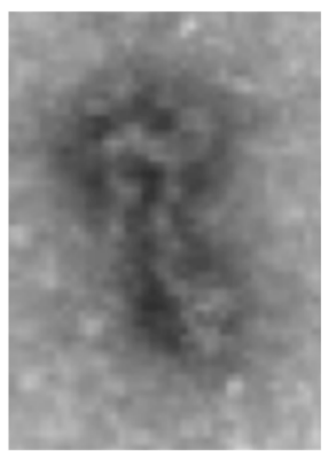

homodimer structure shows that the predicted model fits nicely with the shape determined by EM imaging. Similar observations have been recently obtained for the yeast HP1 proteins, SWI6 [9] 


\section{Implicit Solvation}

A
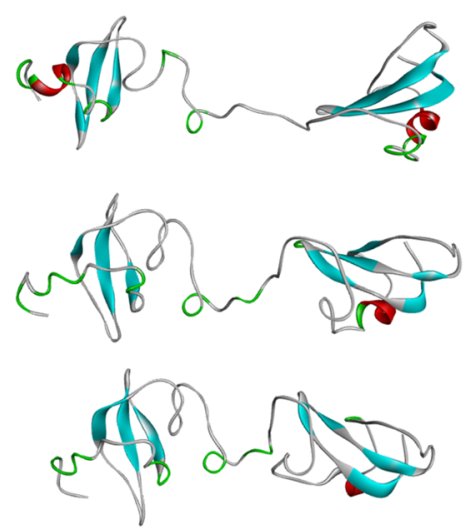

\section{Generalized Born Model}

D
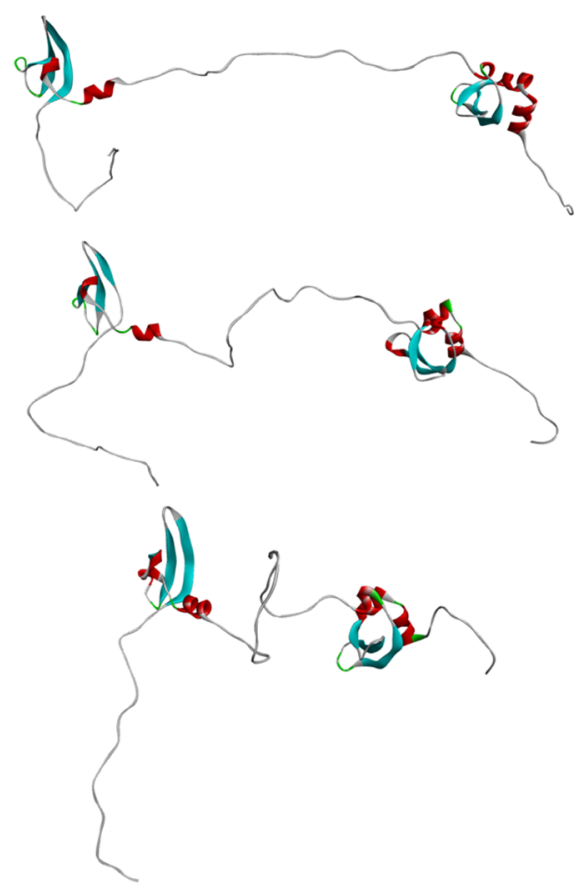

E
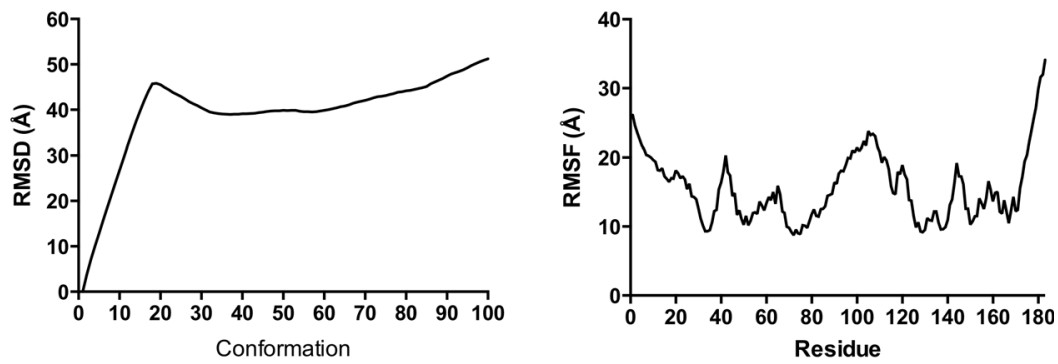

B

C

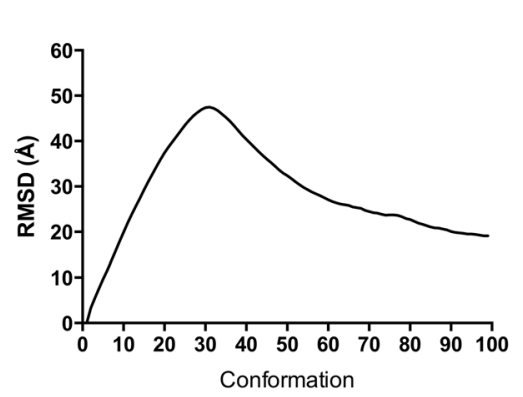

$\mathbf{F}$

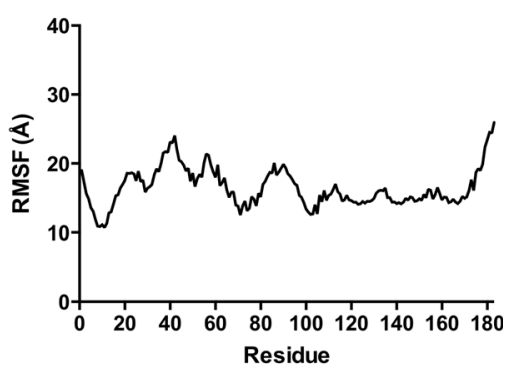

G

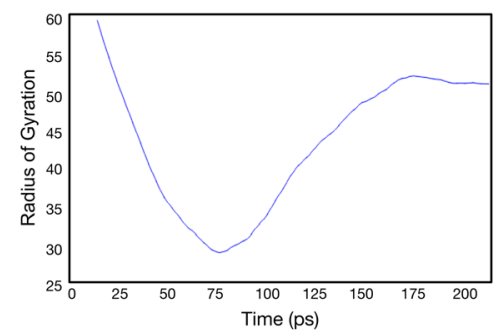

Fig. 4 Molecular dynamics simulations of the HP1 $\gamma$ monomer. In order to gain further insight into the biochemical behavior of HP1 $\gamma$, we subsequently utilized molecular dynamics simulations to perform a conformational search that might reflect the biophysical behavior of HP1 proteins. a An assemblage of conformers obtained during a short 100-ps MD simulation. Analysis of the trajectories obtained through this approach reveals that the highly flexible linker has the ability to shorten the length of this protein very rapidly. b A numerical representation of the flexibility and mobility of this protein during the simulation time was

[62-64], and to recruit different binding partners that regulate chromatin functions [2]. Thus, in summary, molecular modeling reveals HP1 $\gamma$ as an elongated molecule, which in spite of having key globular domains, for the most part behaves as an IDP endowed with a high level of dynamic flexibility. This model is congruent with a significant number of biochemical obtained by calculating the root-mean-square deviation (RMSD) and $\mathbf{c}$ root-mean-square fluctuation (RMSF). d Conformational sampling of the 2-ns GB model simulation highlights the same characteristic flexibility of IDR2 as the implicit solvent model. These results are congruent with the structural bioinformatics analyses, which suggest that the linker region has the highest propensity toward disorder. e, f RMSD and RMSF values for the generalized born (GB) simulation are also represented. $\mathbf{g}$ Radius of gyration calculation for the generalized born (GB) simulation

studies and helps to predict additional experimental data, such as our own electron microscopy data (Fig. 3a-c). Therefore, this model is an attractive tool for studying the molecular behavior of HP1 $\gamma$ in silico, using molecular mechanics and dynamics combined with mutational analyses, different solvation environments, and energetic calculations. 


\section{The IDR2 domain of HP1 $\gamma$ mediates protein-protein interactions: heterodimerization with $\alpha$-importin}

HP1 $\gamma$ has been previously described to be present in the cytoplasm and translocate to the nucleus where it binds to nucleosomes located at promoters [65] and gene bodies [2]. However, how these proteins are transported to the nucleus has not been defined. Functional SLiM prediction algorithms such as PsortII [38] demonstrated that IDR2 HP1 $\gamma$ region primarily forms a bipartite NLS that conforms to the consensus sequence $(\mathrm{K} / \mathrm{R})(\mathrm{K} / \mathrm{R}) X_{10-12}(\mathrm{~K} / \mathrm{R})_{3 / 5}$, where $(\mathrm{K} / \mathrm{R})_{3 / 5}$ represents at least three of either lysine or arginine in five consecutive amino acids (Fig. 5a-b) [66]. Complementary information was gathered by searching PROSITE [67] with the equivalent X-X-X-X-[KRT]-[KA]-R-K-[ST]-X-X-X-X-syntax-based seed, which matched the nuclear localization signal of many known chromatin proteins (Fig. 5a). Experimentally, these results are consistent with results from our proteomic experiments shown in Fig. 5c, in which immunoprecipitation of HP1 $\gamma$ followed by mass spectrometry demonstrated that this protein co-purifies in complex with the NLS receptor proteins, $\alpha$-importins. Thus, we used docking, minimization, and molecular dynamic simulations to develop the first model for an HP1 $\gamma$ - $\alpha$-importin complex, based on the solved structure of the bipartite NLS from nucleophosmin (N1N2). The rules for docking NLS to $\alpha$-importin have been extensively validated by both experimental and modeling studies [32, 68]. Thus, in this study the IDR2 region was modeled first by homology to the conformation described for the isolated N1N2 NLS (PDB: 1PJN), which is a paradigm for docking homologous peptides to $\alpha$-importin [32]. For this purpose, we used the crystal structure of an $\mathrm{N}$-terminal truncated mouse $\alpha$-importin lacking residues $1-69$, as these residues are responsible for autoinhibition. In this model, we observe $\alpha$ importin as a single elongated domain built from ten Arm structural repeats, each containing three $\alpha$ helices $(\mathrm{H} 1, \mathrm{H} 2$, and H3) connected by loops (Fig. 6a). Both the N-terminal and $\mathrm{C}$-terminal basic stretches of amino acids within the HP1 $\gamma$
A

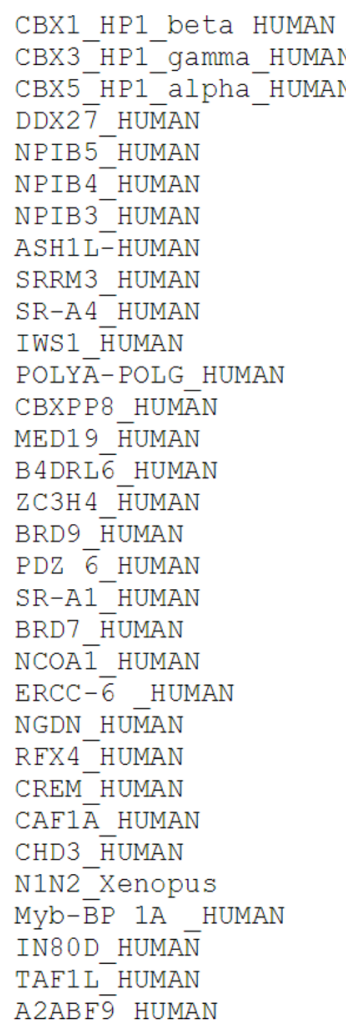

B

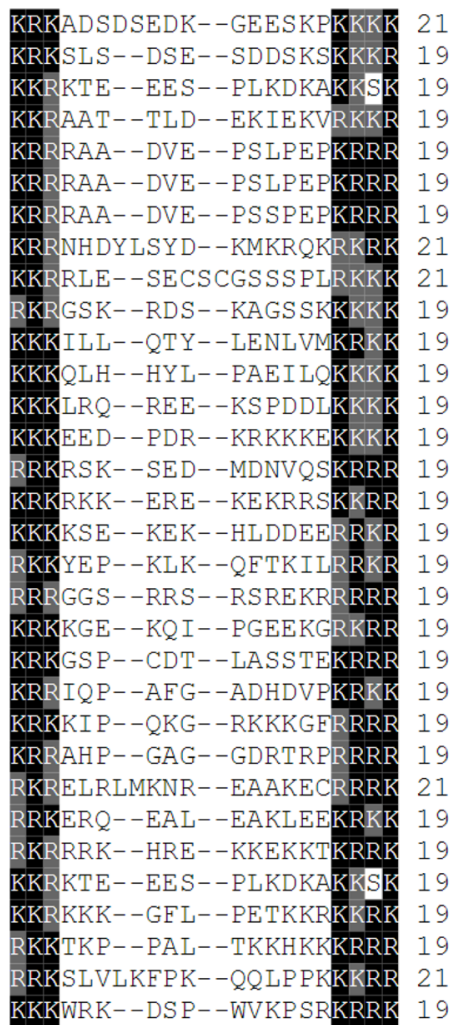

Fig. 5 Identification of a nuclear localization signal in $\mathrm{HP} 1 \gamma$. a Sequence comparison of HP1 NLS motifs with other validated NLS sequences was performed with MUSCLE [76]. This comparison validates the presence of these motifs in these proteins. b Since no mutational analyses exist that provide clues as to the cellular and molecular function of this region of the HP1 proteins, we applied the PsortII algorithm to determine that they primarily form a bipartite NLS that conforms to the consensus sequence $(\mathrm{K} / \mathrm{R})(\mathrm{K} / \mathrm{R}) X 10-12(\mathrm{~K} / \mathrm{R}) 3 / 5$, where $(\mathrm{K} / \mathrm{R}) 3 / 5$ represents at least three of

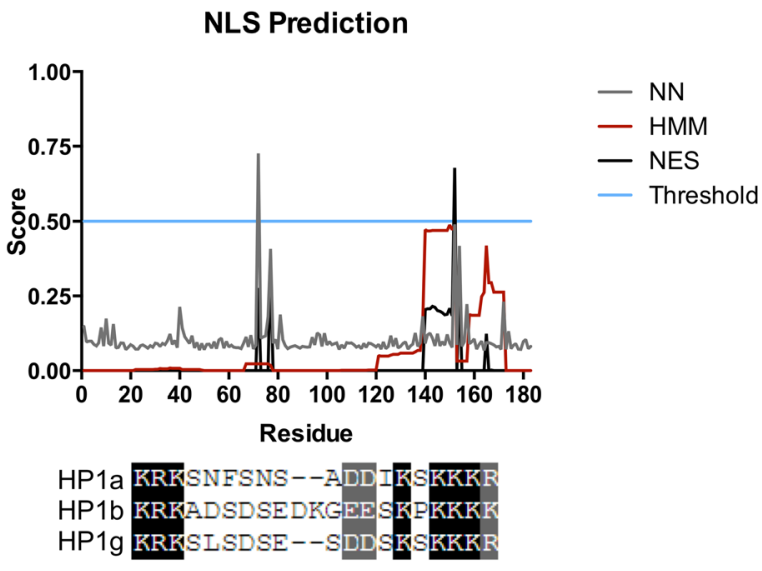

C

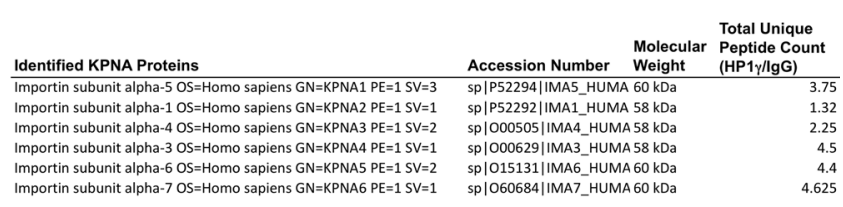

either lysine or arginine of five consecutive amino acids. The fact that the linkers of HP1 proteins are primarily composed of NLS motifs is in agreement with the results of our sequence-to-structural predictions, since all structural studies performed to date for this type of domains reveal their high degree of flexibility and tendency to disorder. c Immunoprecipitation of HP1 $\gamma$ followed by mass spectrometry demonstrated that this protein co-purifies in complex with the NLS receptor proteins, $\alpha$-importins 
linker (IDR2) primarily interact with $\alpha$-importin via salt bridges while the intervening residues additionally contribute to the complex by establishing hydrogen bonds. The two basic clusters of the HP1 $\gamma$ linker bind to two separate well-defined binding sites on the surface of the $\alpha$-importin molecule, referred to as the minor and major sites (Fig. 6a). The minor site specifically binds to the $\mathrm{N}$-terminal basic cluster $\mathrm{KR}$, and the larger, C-terminal basic cluster KRKK binds to the major site. Notably, HP1 $\gamma$ fits nicely within the binding pocket of $\alpha$ importin, with a high complementarity in charge and shape. The steric environments created by this binding mode provide space for both the chromodomain and chromoshadow domain to extend outward and downward from the intermolecular interface (Fig. 6b). The details of these interactions are listed in Supplementary Table 3 and are represented graphically in Fig. 6a-b. We subsequently refined this model by molecular dynamics simulations (Fig. 6c). Compared with the model of an isolated HP $1 \gamma$ molecule, binding of this protein to importin restricts its motion. In addition, compared with the first complex of its isolated NLS, binding of the IDR2 of the full-length HP1 $\gamma$ to $\alpha$-importin is stabilized by additional bonds. Taking

\section{A}

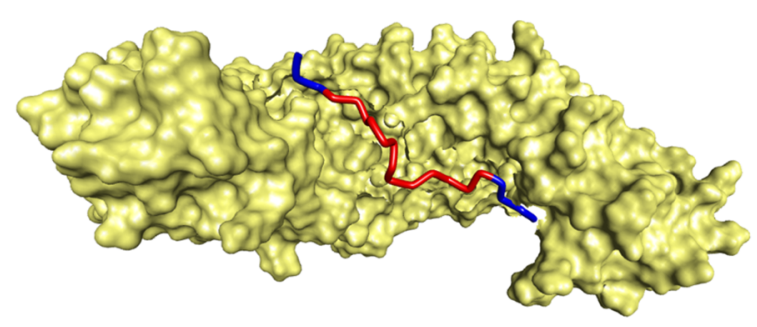

into consideration that, as shown below, this region is amenable to extensive posttranslational modifications, which may interfere or enhance these intermolecular interactions, this model will facilitate future mechanistic understanding of how signaling cascades influence the nuclear transport and thus the function of HP1 $\gamma$ and, by homology, how its other isoforms (HP1 $\alpha$ and HP1 $\beta$ ) as well as its orthologs (e.g., SWI6) are transported.

\section{The IDR1 and IDR2 domains of HP1 $\gamma$ mediate protein-DNA interactions}

Next, we used three different yet complementary approaches to identify residues involved in DNA binding by HP1 $\gamma$ [39]. The first method, DP-bind, implements three machine learning methods - support vector machine (SVM), kernel logistic regression (KLR), and penalized logistic regression (PLR) - to predict DNA-binding and RNA-binding residues from primary structure features, including the side-chain $\mathrm{p} K_{\mathrm{a}}$ value, hydrophobicity index, and molecular mass of an amino acid [39]. Figure 7a provides a graphical representation of the results
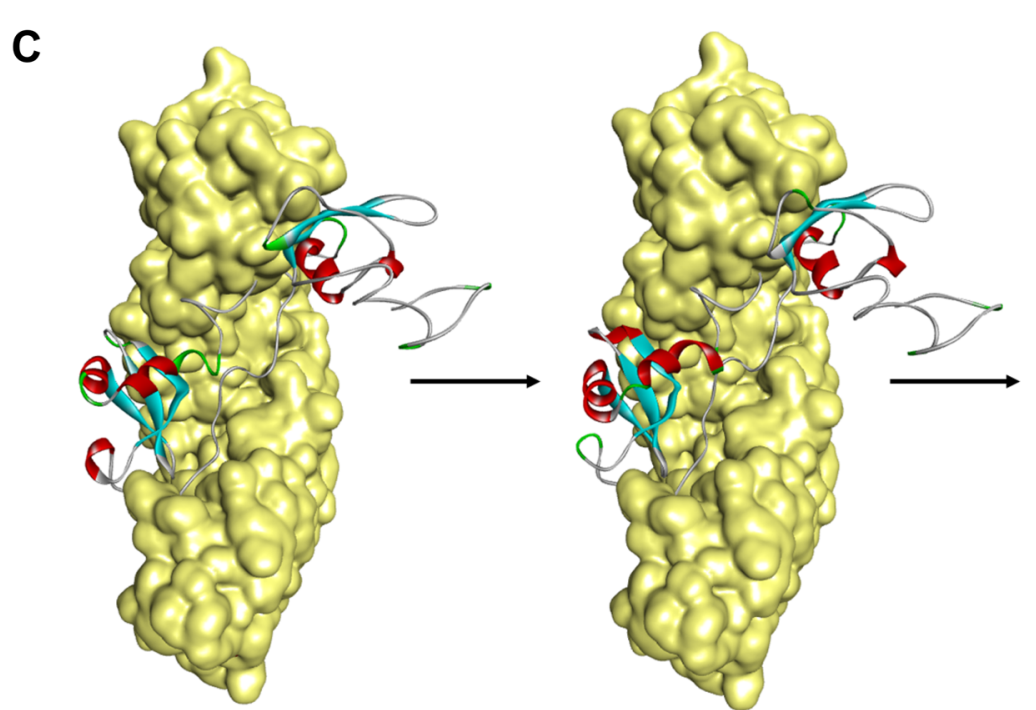

B

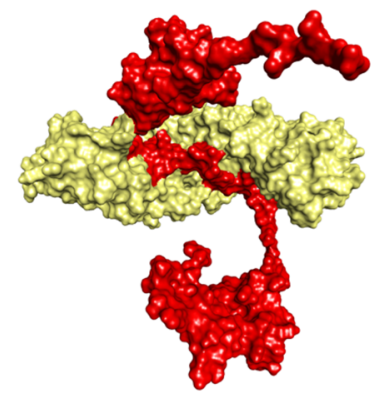

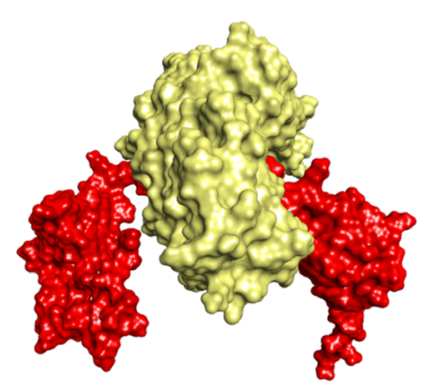

Fig. 6 Modeling and simulation of the HP1 $\gamma$ - $\alpha$-importin complex. a Model of IDR docked to the binding site of $\alpha$-importin. The minor site specifically binds to the N-terminal basic cluster KR (represented in blue), and the larger, C-terminal basic cluster KRKK (represented in blue) binds to the major site. b Model of HP1 $\gamma$ bound to $\alpha$-importin. The steric environments created by this interaction leaves room for both the chromodomain and chromoshadowdomain of HP1 $\gamma$ to extend outward and downward from the intermolecular interphase. c Molecular dynamics simulation of the complex. Unlike the isolated HP1 $\gamma$ simulation, binding to $\alpha$-importin greatly restricts the disordered motion of IDR2 
A

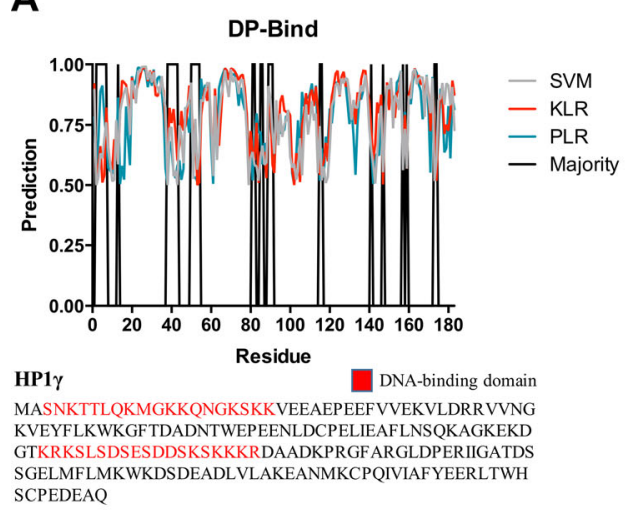

D

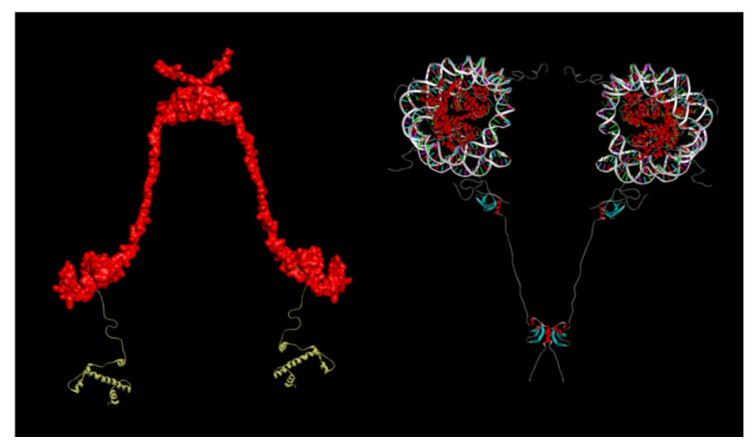

Fig. 7 Modeling of HP1 $\gamma$-DNA complexes. a Prediction of DNAbinding residues by DP-Bind. Results of SVM, KLR, and PLR are represented along with the majority or consensus score of the three predictions, showing DNA-binding residues in the three IDR regions. b HP1 $\gamma$-DNA complex generated by DP-Dock. Note how the interaction of IDR2 with B-DNA is consistent with the results of the DP-Bind

obtained with this approach, which predicted that the two basic clusters (KRKS-( $\left.\mathrm{X}_{9}\right)$-KSKKKR) from the NLS sequence have the potential to interact to DNA. A second basic DNA binding region, present at the most $\mathrm{N}$-terminal region of the protein carrying the sequence SNKTTLQMGKKQNGKSKK was also identified by this method. Interestingly, these predictions are in agreement with experimental data [69], though no details of these interactions currently exist at the atomic resolution level. Subsequently, we sought confirmation of this finding by DPDock, another independent unbiased prediction approach, which uses different rules and 3D structural models as input. An added value of DP-Dock is that, if successful, it would allow the generation of structural HP1 $\gamma$-DNA complexes that can be used for many biochemical and drug-discovery studies. Briefly, DP-Dock uses a nonspecific B-DNA to probe the binding site on a 3D model of a protein that is known to bind DNA but for which the specific amino acid to nucleic acid base contacts are unknown [33]. Given the structure of a DNAbinding protein, the method first automatically generates an ensemble of protein-DNA complexes obtained by rigid-body docking with nonspecific canonical B-DNA molecules with the sequence A10-T10 [33]. Models are subsequently selected by clustering and ranking them according to their DNA-protein
C

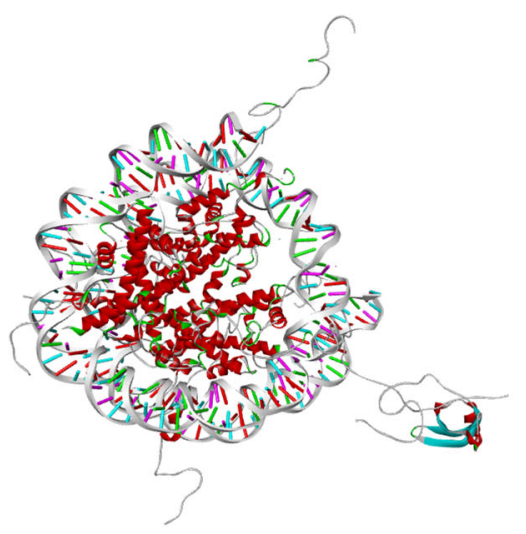

E

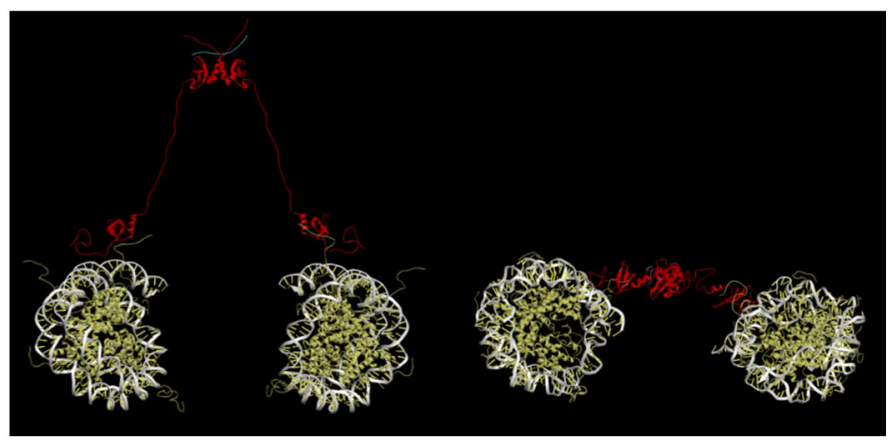

prediction. $\mathbf{c}$ Model of IDR1 bound to a single nucleosome. $\mathbf{d}$ Model of the HP1 $\gamma$ dimer docked to two nucleosomes. e MD simulation of the HP1-nucleosome complex shows the PXVXL-domain docked peptide recruited by the HP1 $\gamma$ dimer. The most $\mathrm{N}$-terminal domain of HP1 $\gamma$ contacts the DNA, which is in agreement with experimental data

interfacial energies [33]. Figure $7 \mathrm{~b}$ demonstrates successful generation of an HP1 $\gamma$-DNA complex where the amino acid to base contacts were primarily given by the same linker region sequence identified through DP-bind (Fig. 7a). Analyses of the protein-DNA interface indicated that residues Asp89, Ser90, Lys91, Ser92, Lys93, Phe105, Gly108, Leu140, Met146, and Lys147 interact with DNA. The ionic and hydrogen-bonding interactions that define the protein-DNA binding interface are listed in Supplementary Table 4. Notably, these residues have been experimentally shown to be involved in DNA binding through the combination of EMSA and site-directed mutagenesis [69]. However, the sequence of DNA used for the DP-dock algorithm is too short for determining whether the N-terminal region of HP1 $\gamma$, as predicted above by DP-BIND (Fig. 7a) and revealed by experimental methods, also interacts with DNA. Consequently, to gain further insight into this phenomenon, we used a third approach based upon carefully examined HP1 $\gamma$ nucleosome models after MD simulations. Noteworthy, we found that following 2 ns of simulation, the dynamic HP1nucleosome contact is characterized by the binding of this $\mathrm{N}$ terminal region of the protein with the DNA duplex. Combined these three methods, DP-BIND, DP-DOCK, and MD simulations of HP1 $\gamma$-nucleosome complexes reveal that $\mathrm{HP} 1 \gamma$ can 
interact with DNA in a sequence-independent manner (Fig. 7ce). Thus, together, these analyses underscore the importance of the intrinsically disordered regions of $\mathrm{HP} 1 \gamma$, not only to support the dynamic behavior of the protein but also to carry information for mediating protein-protein and protein-DNA interactions.

\section{Post-translational modification of the intrinsically disorder regions of HP1 $\gamma$ have the ability to influence intermolecular interactions and histone mimicry}

Since HP1 isoforms function in the regulation of cancerassociated gene expression networks, it is important to gain insight into the mechanisms by which these proteins are either activated or inactivated. A number of histone code-like posttranslational modifications have been described and validated experimentally $[57,58,70]$. To determine other potential posttranslational modification sites that have not been determined, we performed extensive linear motif analyses on HP1 $\gamma$, using the primary sequence as input in order to gain further insight into the differential regulation of this protein. First, posttranslational modifications, such as phosphorylation, acetylation, methylation, ubiquitination, and sumoylation, were predicted using various modification prediction algorithms that create neural networks of potential sites based off a set of experimentally validated sites, support vector machines (SVM), and machine learning methods, such as kernel logistic regression (KLR) and Bayesian decision theory. These sites were then compared to experimentally validated sites listed in PhosphositePlus [57] and PHOSIDA [58] databases. The raw output of these predictions is included in Supplementary Table 5a-e. The results of these analyses are represented graphically in Fig. 8 and reveal that phosphorylation (a), acetylation (b), methylation (c), ubiquitination (d), and sumoylation (e) potentially occur throughout the entire sequence of HP1 $\gamma$. Furthermore, the analyses predicted several potential post-translational modification sites in IDR2 of HP1 $\gamma$ that have not been validated by mass spectrometry, such as phosphorylation sites at Ser70, Ser79, Thr89, and Ser99. Additionally, acetylation sites were predicted at Lys81, Lys84, Lys103, Lys105, Lys107, Lys113; methylation sites were predicted at Lys85, Arg115, Arg119; ubiquitination sites were predicted at Lys81, Lys84, Lys103, Lys 105, Lys107, Lys 113; sumoylation sites were predicted at Arg108 (Fig. 8f). While these sites have not been experimentally validated, they provide additional insight into the differential regulation of HP1 $\gamma$. Since the proteomics experiments were performed on one cell type under one condition, it remains likely that some modification sites from the predicted group integrate signals under conditions that are very different from the culture studies. Thus, both comparisons are justified in this analysis (Fig. 8g). Collectively, the linear motifs present on the linker for nuclear localization, DNA binding, and posttranslational modifications, as well as its predicted propensity
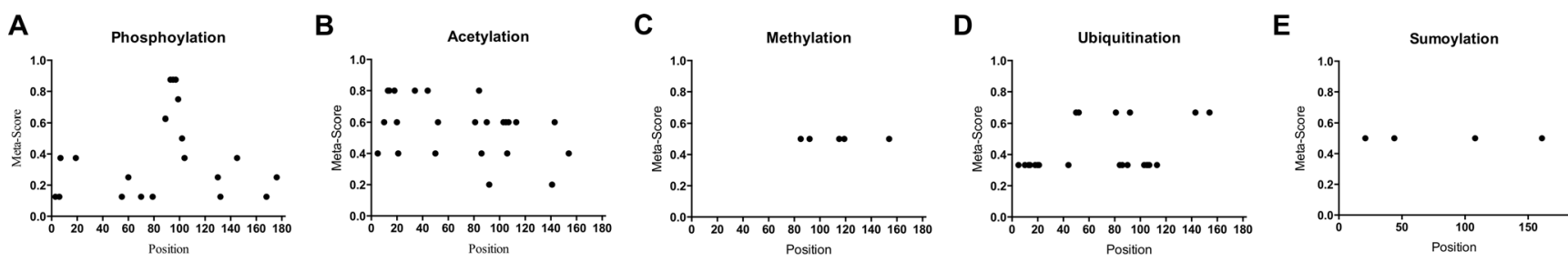

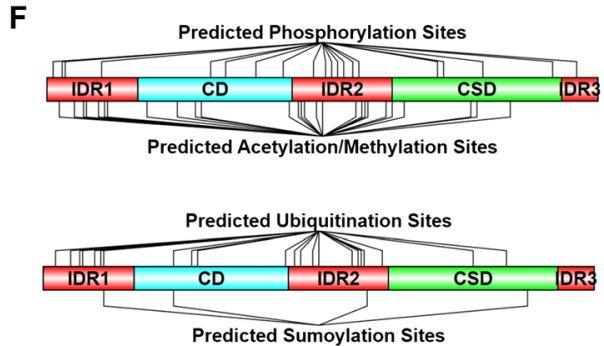

Fig. 8 Characterization of IDR2 as the signal integration center through prediction of post-translational modifications. Prediction of posttranslational modification sites on HP1 $\gamma$ was performed by compiling and statistically scoring linear motifs for phosphorylation (a), acetylation (b), methylation (c), ubiquitination (d), and sumoylation (e) as predicted by 20 different software programs. For each program, we considered sites for which the prediction score was above the cut-off that had been derived using a training set of modified sequences that have been experimentally validated. Subsequently, we developed a metaprediction score by assigning a maximum score of 1 to sites that were

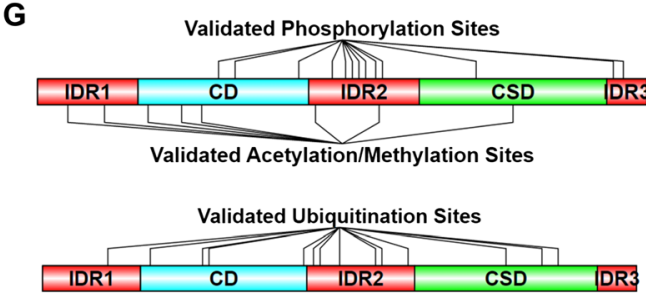

predicted by all of the programs cited. Scores for other programs were numerically expressed relative to this maximum score of 1 . This analysis revealed that predicted phosphorylation sites have high specificity potential near IDR2 of HP1 $\gamma$. f Graphical representation of predicted post-translational modification sites. Results of this analysis revealed that post-translational modifications have the propensity to occur throughout the entire HP1 $\gamma$ sequence, but appear to be heavily localized to the IDR regions. g Graphical representation of experimentally validated post-translational modification sites listed on PhosphositePlus [57] and PHOSIDA [58] 
toward disorder support the hypothesis for the role of IDR2 as the signal integration microdomain for HP1 $\gamma$. In fact, the predicted sites from this analysis prompted us to examine the effects of post-translational modifications and mutations on the biophysical properties of HP1 $\gamma$ and its interactions with some binding partners. For this purpose, we initially constructed a model of a phosphorylated full-length HP1 $\gamma$ as well as its IDR2 and IDR3. We subsequently ran comparative MD simulations of these models in their phosphorylated and unphosphorylated states (Fig. 9a-b). Details of the mutated amino acid positions are provided in the figure legends (Fig. 9). Results of these simulations are consistent with the observation that phosphorylation of IDR 2 restricts HP $1 \gamma$ movement by increasing the time-dependent intramolecular binding (Fig. 9c-d). A similar phenomenon is seen with IDR3, where phosphorylation restricts its movement when compared to the wild-type peptide (Fig. 9e-h). Combined, these results support the relevance of the linear motif analysis by highlighting the biophysical effects of post-translational modifications on the behavior and function of $\mathrm{HP} 1 \gamma$.
In addition, we also analyzed the effect of mutations on the binding affinity between HP1 $\gamma$ and $\alpha$-importin, by performing combinatorial amino acid scanning mutagenesis on residues in IDR2 using MD simulations. The effect of each mutation on the binding energy was calculated by the following equation DDGbind = DG (mutant) - DG (wild type), where DG is the difference between the free energy of the complex and the free energy of the unbound state. Total free energy was calculated using a GB implicit solvent model and is the weighted sum of the van der Waals and electrostatic interactions as described by Spassov and Yan [71]. Briefly, residues in IDR2 were changed to either glutamic acid or alanine: glutamic acid substitutions served as phosphorylationmimicking mutations, while alanine substitutions mimicked non-phosphorylated residues. Calculated mutation energies that were less than $-0.5 \mathrm{kcal} / \mathrm{mol}$ were considered stabilizing while energies greater than $0.5 \mathrm{kcal} / \mathrm{mol}$ were considered destabilizing (Fig. 10). Results of this analysis revealed that phosphorylation-mimicking mutations decreased the affinity of HP1 $\gamma$ for $\alpha$-importin (Fig. 10). Thus, combined, these

\section{Full-Length HP1}

A

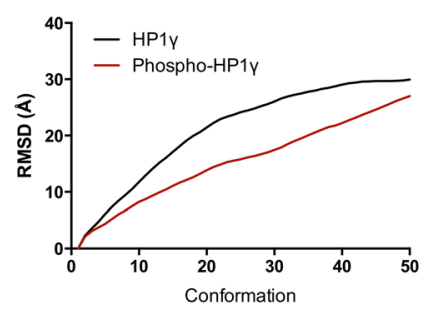

B

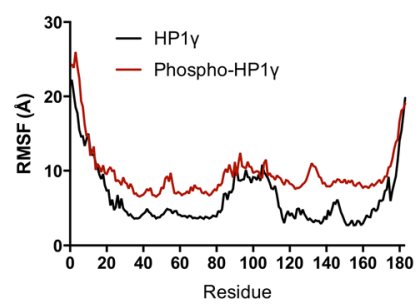

IDR3

E

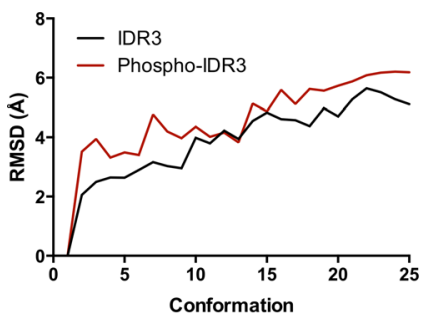

$\mathbf{F}$

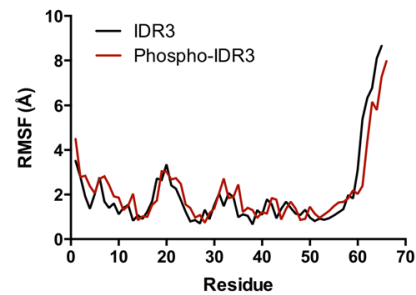

C

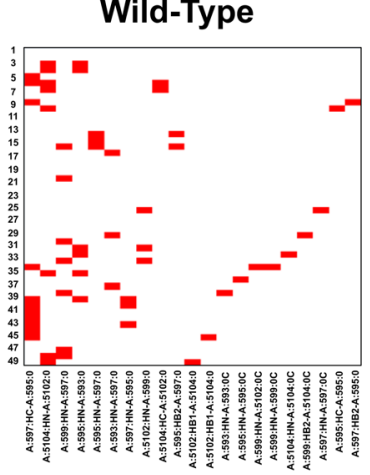

G

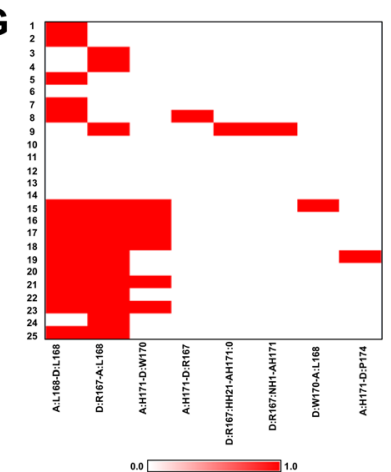

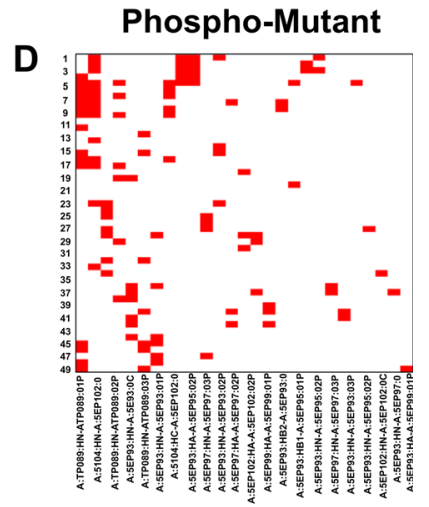

H

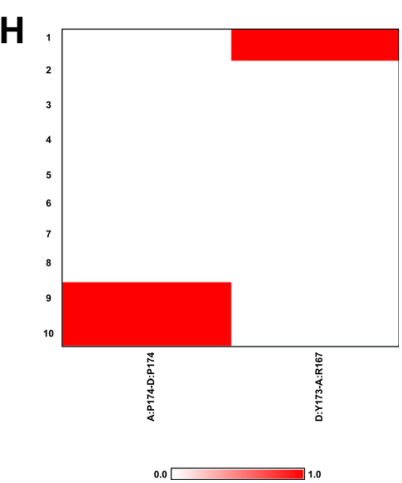

Fig. 9 Effects of mutation on the intramolecular binding properties of HP1 $\gamma$. Molecular dynamics simulations were used to determine the effect of phosphorylation on the stability and intramolecular binding of HP1 $\gamma$. a, b Comparative MD simulations of the full-length wild-type HP1 $\gamma$ and phosphorylated HP1 $\gamma$ with the following amino acid positions mutated: 55, 60, 79, 89, 93, 95, 97, 99, 102, and 176. c, d The simulation results suggest that phosphorylation increases the time-dependent intramolecular binding of the phosphorylated mutant when compared to the wild type. e, f Comparative MD simulations of the full-length wild-type IDR3 and

phosphorylated IDR3 with the following amino acid positions mutated: 169 and 172. g, h Similar to the full-length HP1 $\gamma$, these simulation results suggest that phosphorylation increases the time-dependent intramolecular binding of the phosphorylated mutant when compared to the wild type. Together, these results support the use of linear motif analysis to predict post-translational modifications as these simulations suggest a relevant biophysical effect of phosphorylation on the behavior and intramolecular binding of HP1 $\gamma$ 
Fig. 10 Combinatorial amino acid scanning mutagenesis reveals effect of phosphorylation on the binding affinity between HP $1 \gamma$ and $\alpha$-importin. Residues in IDR2 were changed to either glutamic acid (phosphomimicking) or alanine (nonphosphorylatable). Calculated mutation energies that were less than $-0.5 \mathrm{kcal} / \mathrm{mol}$ were considered stabilizing while energies greater than $0.5 \mathrm{kcal} / \mathrm{mol}$ were considered destabilizing. Phospho-mimicking mutations displayed lower mutation energy profiles and thus a lower binding affinity for $\alpha$-importin. Results of this analysis revealed that phosphorylation-mimicking mutations decrease affinity of HP1 for $\alpha$-importin

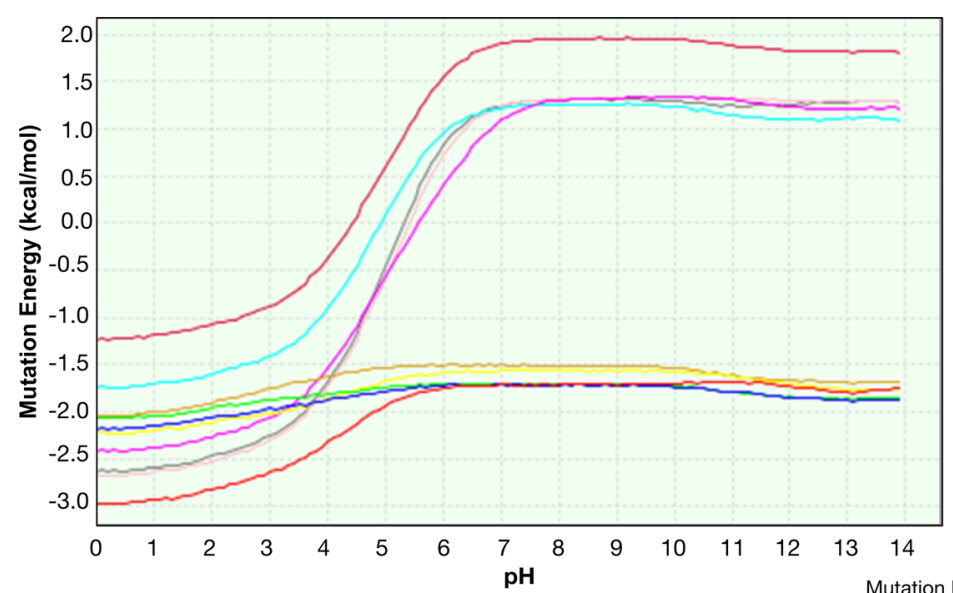

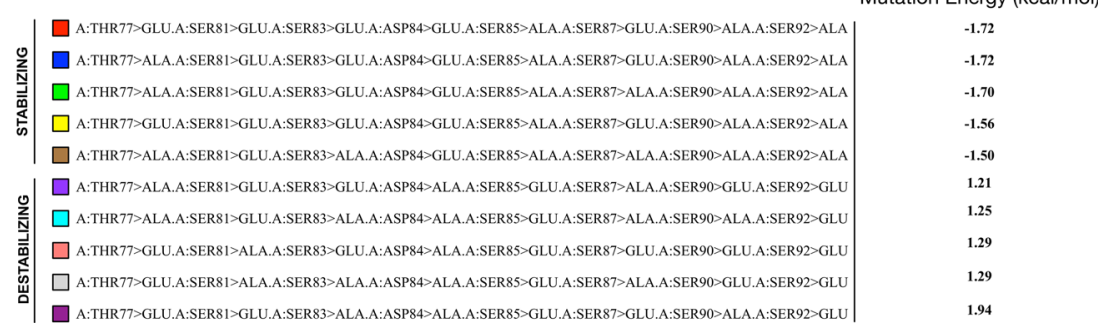

results demonstrate that the dynamic behavior as well as intermolecular interaction properties of HP1 $\gamma$ can be influenced by post-translational modifications, which, because of its solvent accessibility, occur most frequently in the intrinsically disordered regions of this protein.

Lastly, we sought to gain further insight at the atomic level into an observation that has been made by our laboratory and others, which revealed that HP1 $\gamma$ provides a useful example of histone mimicry. Histone mimicry refers to the fact that small linear motifs in non-histone proteins, when appropriately modified, can mimic histone marks and be recognized by histone code writers, readers, and erasers. The structural basis of this phenomenon has also been elucidated for Histone 3 (K9), G9a (K185), and Histone 1.4 (K26) [9, 72]. Though the presence of a histone mimetic peptide within HP1 $\gamma$ (K82) has also been shown biochemically [73], the rules and functional consequences of its interaction with chromodomains remain to be characterized (Fig. 11a). Toward this end, we used a homology-based approach to model the interaction of the HP1 $\gamma$ monomer to its own histone mimicking peptide (Fig. 11b). We modeled the effects that K82 methylation can have on regulating chromodomain-IDR2 interactions. In doing so, we realized that the ability of the HP1 $\gamma$ homodimer to function as a histone mark reader would be inhibited when both of its chromodomains are used to bind to additional monomers, in isolation or as part of another dimer (Fig. 11c). This observation is in agreement with previous biochemical data showing oligomerization of HP1 molecules from yeast, Drosophila, and mammals [9, 62]. More importantly, the ability of HP1 $\gamma$ to autoinhibit through histone mimicry is similar to the behavior of $S$. pombe HP1 (SWI6),

recently derived from elegant kinetic studies [9]. SWI6 also contains a histone-mimicking peptide, though different in sequence and location from that of HP1 $\gamma$. Since the sequence that mediates this event [9] is present in HP1 molecules from many species, this model may help to explain an evolutionarily conserved mechanism for the regulation of this type of histone reader.
A

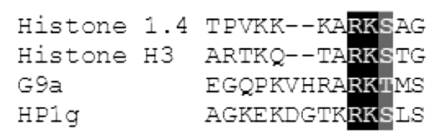

G9a AGKEKDGTKRKSIS

B

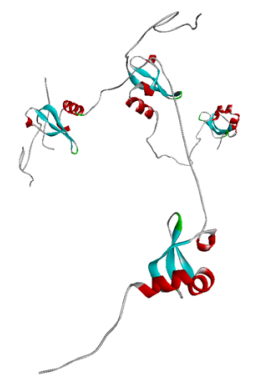

C

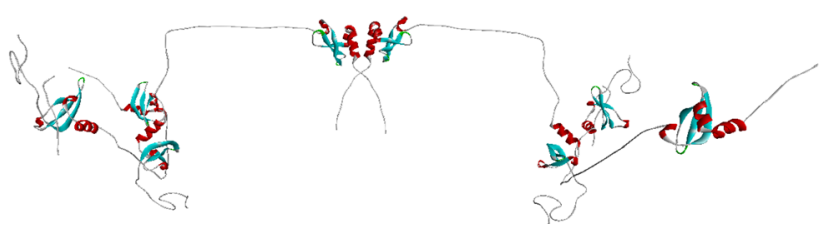

Fig. 11 Modeling of the HP1 $\gamma$ auto-inhibited state. a Sequence alignment of the histone mimetic peptide of HP1 $\gamma$ (K82) with Histone 3 (K9), G9a (K185), and Histone 1.4 (K26). b Homology-based model of HP1 $\gamma$ in its autoinhibited state. c Homology-based model of the autoinhibited HP1 $\gamma$ homodimer. Together, these highlight the hypothesis that the ability of $\mathrm{HP} 1 \gamma$ to function as a histone mark reader is inhibited when both of its chromodomains are used to bind to additional monomers 
In summary, at the onset of the current study, most of the structural considerations related to HP1 $\gamma$ had been confined to its globular chromo and chromoshadow domains. However, little was known about how the rest of the primary sequence influenced the behavior of HP1 $\gamma$. Using several methodologies germane to structural bioinformatics, modeling, docking, dynamics, and mutational analyses, we have gathered evidence that supports a critical contribution of intrinsically disorder regions to define the connectivity, dynamic flexibility, and intermolecular interactions of this protein. This new knowledge, therefore, significantly contributes to further our understanding of the biophysical properties and biochemical behavior of this important epigenetic regulator.

\section{Discussion}

The current work was initiated as a means to extend our understanding of the molecular properties of HP1 $\gamma$. However, based on homology and evolutionary conservation of this protein to other members of its family, our models are likely to be applicable to isoforms and orthologs of HP1 $\gamma$. HP1 proteins are among the most widely characterized epigenetic regulators with many of their functions being conserved throughout evolution [2]. HP1 $\gamma$ associates with the development of human diseases, including many forms of deadly cancers $[3,4]$. Recent studies have applied state-of-the-art biophysical methods to solve the structure of this protein as to advance our understanding of the basic biochemical mechanisms underlying its function and the hope that these efforts will aid in the future design of small drug inhibitors. These studies produced the structure of both the chromodomain and chromoshadow domain $[11,74]$. In spite of this useful information, no reliable full-length model for HP1 $\gamma$ yet exists. Notably, however, extensive biochemical studies indicate that other parts of the protein, namely the most $\mathrm{N}$ - and $\mathrm{C}$-terminal regions as well as the linker, which joins the chromo and chromoshadow domains, may contribute to its function. Toward this end, the current study provides information on the molecular behavior of HP1 $\gamma$ that did not exist before by building and characterizing a structural model for this protein. In fact, our study underscores the critical role of the HP1 $\gamma$ IDRs in molecular connectivity, flexibility, protein-protein, and protein-DNA interactions as well as post-translational modifications, which include histone mimicry. Though useful, HP $1 \alpha$ and HP $1 \beta$ models were also built but not studied in a dynamic fashion, so as to maintain our focus on HP1 $\gamma$. We show models for the HP1 $\gamma$ homodimer (Fig. 2b) and the molecule bound to DNA (Fig 7b-c). We also provide an atomic resolution view of the $\alpha$-importin-HP1 $\gamma$ complex (Fig. 6). We perform, for the first time, an MD simulation of the full-length HP1 $\gamma$ monomer (Fig. 4), in complex with $\alpha$-importin (Fig. 6c), and with nucleosomes (Fig. 7e). These studies indicate that the intrinsically disordered parts of the protein make the human HP1 $\gamma$ protein highly dynamic, a characteristic that had never been previously defined for this protein. Dynamic flexibility given by this region may allow other domains, such as the chromodomain, to more easily sample the tridimensional space in search for binding partners. Thus, we are optimistic that future studies using experimental techniques may test the validity of this interpretation. This dynamic behavior, however, appears to be restricted when HP1 $\gamma$ forms complexes. MD simulations using harmonically restrained nucleosome particles bound by a single HP1 $\gamma$ dimer show that due to its flexibility, it has the potential to recoil onto the nucleosomes. This activity allows for the recruitment of the HP1-binding domain of SUV39H1 through its contact with nucleosomes. Combined, the building and analyses of these structural models provide a more complete description of the biochemical function of HP1 proteins, as elongated molecules with their two globular domains joined by a flexible linker, which endows them with dynamic flexibility and intermolecular recognition properties. Thus, it becomes important to discuss the accuracy, novelty, and mechanistic contribution of this new information to understanding the biochemical properties of these important epigenetic regulators. Several observations are in agreement with and extend, at a predicted atomic resolution, experimentally derived data, increasing the reliability of the models: (1) HP1 $\gamma$ has the ability to form an NLS-importin complex, which renders it competent to translocate into the nucleus (Fig. 5). (2) Once in the nucleus, HP1 $\gamma$ binds to $3 \mathrm{Me}-\mathrm{K} 9 \mathrm{H} 3$ and nucleosomal DNA (Fig. 7). (3) The protein is heavily marked by post-translational modifications, some of them playing a significant role in the regulation of histone mimicry. (4) Similar to its yeast homolog, the histone mimetic peptide within the linker region of HP1 $\gamma$ can be recognized by the chromodomain of this protein, a phenomenon which should inhibit its binding to histone marks. (5) The model suggests that the largest number of post-translational modifications map to the intrinsically disorder regions of the protein, which are more surface exposed. Thus, to our knowledge, when combined, these considerations make the current study novel and important.

Modeling of disordered proteins, such as $\mathrm{HP} 1 \gamma$, is challenging as their structure cannot be represented by a single, derived conformation. These highly flexible molecules sample a multitude of conformations; both expanded and collapsed in nature. Thus, several restrictions were applied in the generation of our model. First, the structure of both the model for the monomer and dimer presented here for HP1 $\gamma$ is in agreement with homology-based data available from structural NMR and SAXS data recently made available for HP1 $\beta$ [61]. This model for the HP1 $\gamma$ monomer complexed nicely with $\alpha$-importin via the IDR2 region, allowing the $\mathrm{N}$-terminal and C-terminal globular domain to protrude out of the complex without steric hindrances (Fig. 6b). Congruently, the 
model of the dimer was built by docking the chromodomain of individual monomers, rather than stitching domains from docked chromodomains. This method leads the N-terminal IDRs and globular domain to adopt a "lobster claw" configuration, which is in agreement with structural data for the highly homologous protein HP1 $\beta$ [75] and yeast SWI6 [9]. It is also true that a single conformation cannot be considered for either the monomer or the dimer. For this reason, we performed conformational sampling using molecular dynamic simulations. Thus, our data is in agreement with the structure of homologous monomers and dimers from human homologues and yeast orthologues, along with their numerous conformations carefully derived from MD simulations, to faithfully represent the structure expected for HP1 $\gamma$. Further modeling studies using longer MD simulations and coarsegrained models may lend more insight into the biochemical behavior or HP1 $\gamma$ and its complexes.

In conclusion, force field-supported, molecular mechanic calculations and analyses of molecular dynamics simulations infer that a significant amount of structure-to-function information is contained within the less studied regions of HP1 $\gamma$. The intrinsically disordered properties of these regions endow the entire molecule with a highly dynamic behavior, intermolecular recognition properties, and the ability to receive signals from several intracellular signaling cascades. Since HP1 $\gamma$ plays a key role in normal epigenetics and cancers, the data and models here reported have current and future applications for better understanding biological and pathobiological functions of this protein. By analogy, this data on HP1 $\gamma$ may also inspire both experimental and in silico testable hypotheses regarding the function of the closest members of this family of proteins.

\begin{abstract}
Acknowledgments This work was supported by funding from the National Institutes of Health (grants R01 CA178627 to GL, R01 DK52913 to RU, R01 AI-089714 to W. A. Faubion, and T32 GM007337 to GV), the Mayo Foundation, as well as the Mayo Clinic Center for Cell Signaling in Gastroenterology (P30DK084567) and the Mayo Clinic SPORE in Pancreatic Cancer (P50 CA102701).
\end{abstract}

Open Access This article is distributed under the terms of the Creative Commons Attribution 4.0 International License (http:// creativecommons.org/licenses/by/4.0/), which permits unrestricted use, distribution, and reproduction in any medium, provided you give appropriate credit to the original author(s) and the source, provide a link to the Creative Commons license, and indicate if changes were made.

\section{References}

1. Eissenberg JC, James T, Foster-Hartnett DM, Hartnett T, Ngan V, Elgin SC (1990) Mutation in a heterochromatin-specific chromosomal protein is associated with suppression of position-effect variegation in Drosophila melanogaster. Proc Natl Acad Sci U S A 87: 9923-9927

2. Lomberk G, Wallrath L, Urrutia R (2006) The Heterochromatin Protein 1 family. Genome Biol 7(7):228
3. Velez G, Urrutia R, Lomberk G (2013) Critical role of the HP1histone methyl transferase pathways in cancer epigenetics. Med Epigenet 1(1):100-105

4. Dialynas GK, Vitalini M, Wallrath LL (2008) Linking Heterochromatin Protein 1 (HP1) to cancer progression. Mutat Res 647(1-2):13-20

5. Bannister AJ, Zegerman P, Partridge JF, Miska EA, Thomas JO, Allshire RC, Kouzarides T (2001) Selective recognition of methylated lysine 9 on histone H3 by the HP1 chromo domain. Nature 410:120-124

6. Lachner M, O'Carroll D, Rea S, Mechtler K, Jenuwein T (2001) Methylation of histone $\mathrm{H} 3$ lysine 9 creates a binding site for HP1 proteins. Nature 410:116-120

7. Daujat $\mathrm{S}$ et al (2005) HP1 binds specifically to Lys26-methylated histone H1.4, whereas simultaneous Ser27 phosphorylation blocks HP1 binding. J Biol Chem 280(45):38090-38095

8. Aasland R, Stewart A (2005) The chromo shadow domain, a second chromo domain in heterochromatin-binding protein 1, HP1. Nucleic Acids Res 23:3168-3173

9. Canzio D et al (2013) A conformational switch in HP1 releases auto-inhibition to drive heterochromatin assembly. Nature 496(7445):377-381

10. Lomberk $G$ et al (2012) Sequence-specific recruitment of heterochromatin protein 1 via interaction with Krüppel-like factor 11, a human transcription factor involved in tumor suppression and metabolic diseases. J Biol Chem 287(16):13026-13039

11. Brasher S et al (2000) The structure of mouse HP1 suggests a unique mode of single peptide recognition by the shadow chromo domain dimer. EMBO J 19(7):1587-1597

12. Thiru A et al (2004) Structural basis of HP1/PXVXL motif peptide interactions and HP1 localisation to heterochromatin. EMBO J 23(3):489-499

13. Ishida T, Kinoshita K (2007) PrDOS: prediction of disordered protein regions from amino acid sequence. Nucl Acids Res 35(Web Server Issue):W460-W464

14. Ishida T, Kinoshita K (2008) Prediction of disordered regions in proteins based on the meta approach. Bioinformatics 24(11): $1344-1348$

15. Hirose $\mathrm{S}$ et al (2007) POODLE-L: a two-level SVM prediction system for reliably predicting long disordered regions. Bioinformatics 23(16):2046-2053

16. Cheng J, Sweredoski M, Baldi P (2005) Accurate prediction of protein disordered regions by mining protein structure data. Data Min Knowl Disc 11(3):213-222

17. Linding $\mathrm{R}$ et al (2003) Protein disorder prediction: implications for structural proteomics. Structure 11(11):1453-1459

18. Dosztanyi $Z$ et al (2005) IUPred: web server for the prediction of intrinsically unstructured regions of proteins based on estimated energy content. Bioinformatics 21(16):3433-3434

19. Xue B et al (2010) PONDR-FIT: a meta-predictor of intrinsically disordered amino acids. Biochim Biophys Acta 1804(4):996-1010

20. Deng X, Eickholt J, Cheng J (2009) PreDisorder: ab initio sequence-based prediction of protein disordered regions. BMC Bioinf 10:436

21. Wang L, Sauer U (2008) OnD-CRF: predicting order and disorder in proteins using [corrected] conditional random fields. Bioinformatics 24(11):1401-1402

22. Yang $\mathrm{Z}$ et al (2005) RONN: the bio-basis function neural network technique applied to the detection of natively disordered regions in proteins. Bioinformatics 21(16):3369-3376

23. Prilusky J et al (2005) FoldIndex: a simple tool to predict whether a given protein sequence is intrinsically unfolded. Bioinformatics 21(16):3435-3438

24. McGuffin L (2008) Intrinsic disorder prediction from the analysis of multiple protein fold recognition models. Bioinformatics 24(16): 1798-1804 
25. Linding R et al (2003) GlobPlot: exploring protein sequences for globularity and disorder. Nucleic Acids Res 31(13):3701-3708

26. Wu S, Zhang Y (2007) LOMETS: a local meta-threading-server for protein structure prediction. Nucleic Acids Res 35(10):3375-3382

27. Wu S, Zhang Y (2008) MUSTER: improving protein sequence profile-profile alignments by using multiple sources of structure information. Proteins 72(2):547-556

28. Sali A, Blundell T (1993) Comparative protein modelling by satisfaction of spatial restraints. J Mol Biol 234(3):779-815

29. Inc AS (2012) Discovery studio modeling environment, release 3.5, in Accelrys Discovery Studio. Accelrys Software Inc, San Diego

30. Willard L et al (2003) VADAR: a web server for quantitative evaluation of protein structure quality. Nucleic Acids Res 31(13):33163319

31. Voss N, Gerstein M (2010) 3V: cavity, channel and cleft volume calculator and extractor. Nucleic Acids Res 38(Web Server Issue): W555-W562

32. Fontes M et al (2003) Structural basis for the specificity of bipartite nuclear localization sequence binding by importin-alpha. J Biol Chem 278:27981-27987

33. Gao M, Skolnick J (2009) From nonspecific DNA-protein encounter complexes to the prediction of DNA-protein interactions. PLoS Comput Biol 5(3):e1000341

34. Urrutia R et al (2014) Evidence supporting the existence of a NUPR1-like family of helix-loop-helix chromatin proteins related to, yet distinct from, AT hook-containing HMG proteins. J Mol Model 20(8):1-20

35. Cornell WD, Cieplak P, Bayly CI, Gould IR, Merz KM, Ferguson DM, Spellmeyer DC, Fox T, Caldwell JW, Kollman PA (1995) A second-generation force field for the simulation of proteins, nucleic acids, and organic molecules. J Am Chem Soc 117:5179-5197

36. Ryckaert JP, Ciccotti G, Berendsen HJC (1977) Numerical integration of the Cartesian equations of motion of a system with constraints: molecular dynamics of $n$-alkanes. J Comput Phys 23(3): 327-341

37. Im W, Lee M, Brooks C (2003) Generalized born model with a simple smoothing function. J Comput Chem 24(14):1692-1702

38. Nakai K, Horton P (1999) PSORT: a program for detecting sorting signals in proteins and predicting their subcellular localization. Trends Biochem Sci 24(1):34-36

39. Hwang S, Gou Z, Kuznetsov IB (2007) DP-Bind: a web server for sequence-based prediction of DNA-binding residues in DNAbinding proteins. Bioinformatics 23(5):634-636

40. Blom N, Sicheritz-Ponten T, Gupta R, Gammeltoft S, Brunak S (2004) Prediction of post-translational glycosylation and phosphorylation of proteins from the amino acid sequence. Proteomics 4(6): 1633-1649

41. Wong YH, Lee T, Liang HK, Huang CM, Wang TY, Yang YH, Chu CH, Huang HD, Ko MT, Hwang JK (2007) KinasePhos 2.0: a web server for identifying protein kinase-specific phosphorylation sites based on sequences and coupling patterns. Nucleic Acids Res 35: W588-W594

42. Iakoucheva LM, Radivojac P, Brown CJ, O'Connor TR, Sikes JG, Obradovic Z, Dunker AK (2004) The importance of intrinsic disorder for protein phosphorylation. Nucleic Acids Res 32(3):10371049

43. Dou Y, Yao B, Zhang C (2014) PhosphoSVM: prediction of phosphorylation sites by integrating various protein sequence attributes with a support vector machine. Amino Acids 46(6):1459-1469

44. Gao J, Thelen J, Dunker AK, Xu D (2010) Musite, a tool for global prediction of general and kinase-specific phosphorylation sites. Mol Cell Proteomics 9(12):2586-2600

45. Xue Y, Li A, Wang L, Feng H, Yao X (2006) PPSP: prediction of PK-specific phosphorylation site with Bayesian decision theory. BMC Bioinf 7:163
46. Li A, Xue Y, Jin C, Wang M, Yao X (2006) Prediction of Neacetylation on internal lysines implemented in Bayesian Discriminant Method. Biochem Biophys Res Commun 350(4): 818-824

47. Wang L, Du Y, Lu M, Li T (2012) ASEB: a web server for KATspecific acetylation site prediction. Nucleic Acids Res 40:W376W379

48. Shao J, Xu D, Hu L, Kwan YW, Wang Y, Kong X, Ngai SM (2012) Systematic analysis of human lysine acetylation proteins and accurate prediction of human lysine acetylation through bi-relative adapted binomial score Bayes feature representation. Mol BioSyst 8(11):2964-2973

49. Li S, Li H, Li M, Shyr Y, Xie L, Li Y (2009) Improved prediction of lysine acetylation by support vector machines. Protein Pept Lett 16(8):977-983

50. Hou T et al (2014) LAceP: lysine acetylation site prediction using logistic regression classifiers. PLoS ONE 9(2):e89575

51. Shao J, Xu D, Tsai SN, Wang Y, Ngai SM (2009) Computational identification of protein methylation sites through bi-profile Bayes feature extraction. PLoS ONE 4(3):e4920

52. Shein D et al (2009) Incorporating structural characteristics for identification of protein methylation sites. J Comput Chem 30(9): $1532-1543$

53. Li A, Gao X, Ren J, Jin C, Xue Y (2009) BDM-PUB: computational prediction of protein ubiquitination sites with a Bayesian discriminant method

54. Chen Z, Chen Y, Wang X, Wang C, Yan R, Zhang Z (2011) Prediction of protein ubiquitination sites by using the composition of k-spaced amino acid pairs. PLoS ONE 6(7):e22930

55. Radivojac P, Vacic V, Haynes C, Cocklin RR, Mohan A, Heyen JW, Goebl MG, Iakoucheva LM (2010) Identification, analysis and prediction of protein ubiquitination sites. Proteins 78(2):365-380

56. Zhao Q, Xie Y, Zheng Y, Jiang S, Liu W, Mu W, Zhao Y, Xue Y, Ren J (2014) GPS-SUMO: a tool for the prediction of sumoylation sites and SUMO-interaction motifs. Nucleic Acids Res 42(W1): W325-W330

57. Hornbeck PV, Kornhauser J, Tkachev S, Zhang B, Skrzypek E, Murray B, Latham V, Sullivan M (2012) PhosphoSitePlus: a comprehensive resource for investigating the structure and function of experimentally determined post-translational modifications in man and mouse. Nucleic Acids Res 40(Database issue):D261-D270

58. Gnad F, Gunawardena J, Mann M (2011) PHOSIDA 2011: the posttranslational modification database. Nucleic Acids Res 39(Database Issue):D253-D260

59. Wong E, Na D, Gsponer J (2013) On the importance of polar interactions for complexes containing intrinsically disordered proteins. PLoS Comput Biol 9(8):e1003192

60. Laskowski RA, MacArthur MW, Moss DS, Thornton JM (1993) PROCHECK - a program to check the stereochemical quality of protein structures. J Appl Crystallogr 26:283-291

61. Munari F et al (2014) Characterization of the effects of phosphorylation by CK2 on the structure and binding properties of human HP1 $\beta$. FEBS Lett 588(7):1094-1099

62. Canzio D et al (2011) Chromodomain-mediated oligomerization of HP1 suggests a nucleosome-bridging mechanism for heterochromatin assembly. Mol Cell 41:67-81

63. Munari $\mathrm{F}$ et al (2012) Methylation of lysine 9 in histone $\mathrm{H} 3$ directs alternative modes of highly dynamic interaction of heterochromatin protein hHP1 $\beta$ with the nucleosome. J Biol Chem 287(40):3375633765

64. Ruthenburg A et al (2007) Multivalent engagement of chromatin modifications by linked binding modules. Nat Rev Mol Cell Biol 8(12):983-994

65. Smothers J, Henikoff S (2001) The hinge and chromo shadow domain impart distinct targeting of HP1-like proteins. Mol Cell Biol 21(7):2555-2569 
66. Kosugi S et al (2009) Six classes of nuclear localization signals specific to different binding grooves of importin $\alpha$. J Biol Chem 284:478-485

67. Sigrist CJ, Cerutti L, de Castro E, Langendijk-Genevaux PS, Bulliard V, Bairoch A, Hulo N (2010) PROSITE, a protein domain database for functional characterization and annotation. Nucleic Acids Res 38:D161-D166

68. Fontes M, Teh T, Kobe B (2000) Structural basis of recognition of monopartite and bipartite nuclear localization sequences by mammalian importin-alpha. J Mol Biol 297(15):1183-1194

69. Zhao $\mathrm{T}$ et al (2000) Heterochromatin protein 1 binds to nucleosomes and DNA in vitro. J Biol Chem 275(36):28332-28338

70. Lomberk G, Bensi D, Fernandez-Zapico ME, Urrutia R (2006) Evidence for the existence of an HP1-mediated subcode within the histone code. Nat Cell Biol 8:407-415
71. Spassov V, Yan L (2008) A fast and accurate computational approach to protein ionization. Protein Sci 17(11):1955-1970

72. Sampath S et al (2007) Methylation of a histone mimic within the histone methyltransferase G9a regulates protein complex assembly. Mol Cell 27(4):596-608

73. Papamokos G et al (2012) Structural role of RKS motifs in chromatin interactions: a molecular dynamics study of HP1 bound to a variably modified histone tail. Biophys J 102(8):1926-1933

74. Jacobs S, Khoransanizadeh S (2002) Structure of HP1 chromodomain bound to a lysine 9-methylated histone $\mathrm{H} 3$ tail. Science 295(5562):2080-2083

75. Munari F et al (2013) Structural plasticity in human heterochromatin protein $1 \beta$. PLoS ONE 8(4):e60887

76. Edgar R (2004) MUSCLE: multiple sequence alignment with high accuracy and high throughput. Nucleic Acids Res 32:1792-1797 